\title{
Fine-grained Information Flow Analysis for a $\lambda$-calculus with Sum Types
}

\author{
Vincent Simonet \\ INRIA Rocquencourt \\ E-mail: Vincent.Simonet@inria.fr
}

\begin{abstract}
This paper presents a new type system tracing information flow for a $\lambda$-calculus equipped with polymorphic "let" and with sums (a.k.a. union types or polymorphic variants). The type system allows establishing (weak) noninterference properties. Thanks to original forms of security annotations and constraints, it is more accurate than existing analyses. Through a straightforward encoding into sums, this work also provides a new type-based information flow analysis for programming languages featuring exceptions. From these systems, one may derive constraint-based formulations, in the style of $H M(X)$, which have decidable type inference.
\end{abstract}

\section{Introduction}

Information flow analysis is concerned with statically determining the dependencies between the inputs and the outputs of a program. It allows establishing instances of a non-interference property that may address secrecy and integrity issues.

Although the first pieces of work in this area appear in the late 70's [2], such an analysis has been formulated as a type system only in the past few years $[8,18,3,12]$. Types seem to be most suitable for static analysis of information flow. Practically speaking, they may serve as a specification langage, offer automated verification of code - provided type inference is available - and have no run-time cost. From a theoretical point of view, they can express non-interference results in simple and precise ways.

In these systems, types are usually annotated with security levels chosen in a suitable lattice. Each annotation gives an approximation of the information which the expression that it describes may convey. Thus type constructors for (expressions producing) base values - e.g. integers or enumerated constants - carry one security level representing all of the information attached to the value. Such an approximation may be too restrictive in some cases. Indeed, consider the program fragment

$$
\begin{array}{r}
\text { let } t=\text { if } x \text { then (if } y \text { then } A \text { else } B \text { ) } \\
\text { else (if } z \text { then } A \text { else } D \text { ) }
\end{array}
$$

(Here, the language is equipped with three constants $A, B$, $D$ belonging to the same datatype.) $t$ 's value directly depends on the booleans $x, y$ and $z$. Previous type systems will record this potential information flow by constraining the security level attached to $t$ to exceed those of $x, y$ and $z$. As a result, they will similarly constrain the level attached to the integer $u$ produced by

$$
\text { let } u=t \text { case }[A, B \mapsto 1 \mid D \mapsto 0]
$$

Yet, $u$ does not depend on $y$ because testing whether $t$ 's value is $D$ rather than $A$ or $B$ cannot leak any information about $y$.

Our work is a proposal for a more sophisticated analysis (concerning information flow) of sum types. In the previous example, we will attach a (triangular) matrix of three security levels $q(A \cdot B), q(B \cdot D)$ and $q(A \cdot D)$ to the identifier $t$ : one for each (unordered) pair of constructors. Thus $q(A \cdot B)$ describes how much information one may leak by testing whether $t$ 's value is $A$ rather than $B$. It must therefore be at least the union of $x$ and $y$ 's levels. Similarly, $q(A \cdot D)$ must be the union of $x$ and $z$ 's levels and $q(B \cdot D)$ must be $x$ 's level or greater. In the previous example, because the test allows to determine whether $t$ 's value is $D$ rather than $A$ or $B$, our system will approximate the possible information leak by $q(A \cdot D) \sqcup q(B \cdot D)$, i.e. the union of $x$ and $z$ 's levels. Therefore, it will be able to establish the absence of dependency between $y$ and $u$.

Recent studies in the area of information flow analysis concern realistic programming languages providing an exception mechanism - such as Java [5] or ML [13]. The treatment of exceptions in these systems seems relatively ad hoc and is not perfectly well understood: although there exists a simple monadic encoding of exceptions into sums $[4,19]$, these systems address exceptions in a direct manner. Indeed, both [5] and [13] try to achieve a better precision in the analysis of information flow due to exceptions than existing systems [3] dealing with sums provide. 
Because we describe a very accurate type system for sums, we are able to obtain a suitable analysis for exceptions by a simple translation. There are two reasons why this approach is interesting. First, it describes a new analysis of information flow due to exceptions that is more accurate than existing ones. Second, because it may encode existing systems, it allows a better understanding of their design.

\section{Overview}

In the current paper, we follow a similar approach to that developed in [13] for Core ML. Section 3 introduces the language $\lambda_{+}$and a technical extension, called $\lambda_{+}^{2}$, that allows us to reason about the simultaneous reduction of two expressions. We present in section 4 the type system and state the non-interference theorem. Then we address the question of type inference in section 5 and present a small set of examples in section 6. Section 7 explains how it is possible to obtain from the type system for sums another one for exceptions by a simple translation of the latter in the former. Lastly, section 8 proposes some restrictions of these systems and relates them to existing work.

By lack of space, some proofs are omitted; they can be found in the full version of this paper [16].

\section{$3 \lambda$-calculus with sums}

Throughout this paper, every occurence of $*$ stands for a distinct anonymous meta-variable of appropriate kind.

\subsection{The $\lambda_{+}$-calculus}

Let $k$ range over integers; let $x, c$ range over two disjoint, denumerable sets of program variables and constructor names, respectively. We denote by $\mathcal{C}$ the set of all constructor names and let $C$ range over subsets of $\mathcal{C}$. (In the examples, we will assume $\{A, B, D\} \subseteq \mathcal{C}$.) Then, expressions and handlers are defined as follows:

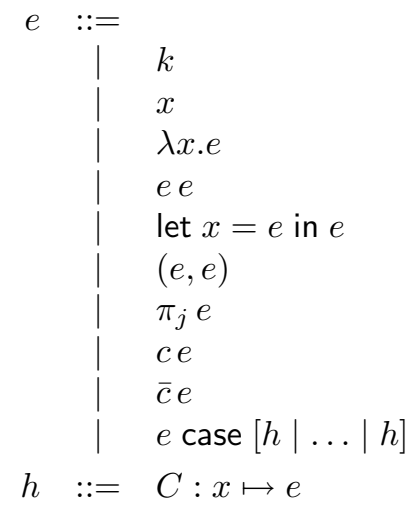

expression (integer constant) (program variable) (abstraction) (application)

(local definition) (pair construction) (pair projection, $j \in\{1,2\}$ ) (sum construction) (sum destruction) (sum case) handler
Our language includes the $\lambda$-calculus with pairs and a "let" binding in the style of ML. It is extended with sum expressions built by the construction $c e$. For each constructor name $c, \lambda_{+}$provides a destructor $\bar{c}$ such that $\bar{c}(c e)$ evaluates to $e$. A handler is a triple of a subset of $\mathcal{C}$, a program variable and an expression. The case construction allows to match an expression's head constructor against a list of handlers: $c e$ case $\left[C_{1}: x_{1} \mapsto e_{1}|\ldots| C_{n}: x_{n} \mapsto e_{n}\right]$ reduces to $e_{j}\left[x_{j} \Leftarrow c e\right]$ if $c \in C_{j}$. (In the following, we will assume that case expressions are deterministic, i.e. that the $C_{j}$ are pairwise disjoint.) The conjunction of projections and case clauses gives us the expressiveness of $a$ la $M L$ pattern matching on data constructors. We still provide specific integer constants, although they may be considered as a special case of sums, because they help state non-interference theorems in a concise manner. Lastly, we do not provide any construction allowing recursion; but this can be achieved straightforwardly, for instance by introducing an extra parameter $f$ in the $\lambda$ construct as in [13].

\subsection{The $\lambda_{+}^{2}$ calculus}

Because establishing a non-interference result requires reasoning about two expressions and exhibiting a bisimulation between their executions, we introduce a technical extension of $\lambda_{+}$. It allows to deal simultaneously with two expressions that share some sub-terms throughout a reduction.

This extension, called $\lambda_{+}^{2}$, is similar to Core $\mathrm{ML}^{2}$ in [13]. It is as follows:

$$
e \quad:=\ldots \mid\langle e \mid e\rangle
$$

We do not allow nesting $\langle\cdot \mid \cdot\rangle$ constructs. A $\lambda_{+}^{2}$ term represents a pair of $\lambda_{+}$terms. For instance, the $\lambda_{+}^{2}$ expression $\left\langle e_{1} \mid e_{2}\right\rangle$ encodes the pair $\left(e_{1}, e_{2}\right)$. Because brackets can appear at an arbitrary depth within a $\lambda_{+}^{2}$ term, the encoding allows to keep track of sharing: assuming $e, e_{1}$ and $e_{2}$ are $\lambda_{+}$expressions, both $\left\langle e_{1} \mid e_{2}\right\rangle e$ and $\left\langle e_{1} e \mid e_{2} e\right\rangle$ encode the pair $\left(e_{1} e, e_{2} e\right)$, but the former explicitly records the fact that the argument $e$ is shared.

In order to relate a $\lambda_{+}^{2}$ term to the pair of $\lambda_{+}$expressions which it encodes, we define two projections, $\lfloor\cdot\rfloor_{1}$ and $\lfloor\cdot\rfloor_{2}$. They are homomorphisms except at $\langle\cdot \mid \cdot\rangle$ nodes: $\left\lfloor\left\langle e_{1}\right|\right.$ $\left.\left.e_{2}\right\rangle\right\rfloor_{i}=e_{i}$. We extend them to handlers by $\lfloor C: x \mapsto e\rfloor_{i}=$ $C: x \mapsto\lfloor e\rfloor_{i}$ and pointwise to lists of handlers $\vec{h}$.

The capture-free substitution of $v$ for $x$ in $e$, written $e[x \Leftarrow v]$, is defined in the usual way, except at $\langle\cdot \mid \cdot\rangle$ nodes, where we must use an appropriate projection of $v$ in each branch: $\left\langle e_{1} \mid e_{2}\right\rangle[x \Leftarrow v]$ is $\left\langle e_{1}\left[x \Leftarrow\lfloor v\rfloor_{1}\right]\right| e_{2}[x \Leftarrow$ $\left.\left.\lfloor v\rfloor_{2}\right]\right\rangle$. 


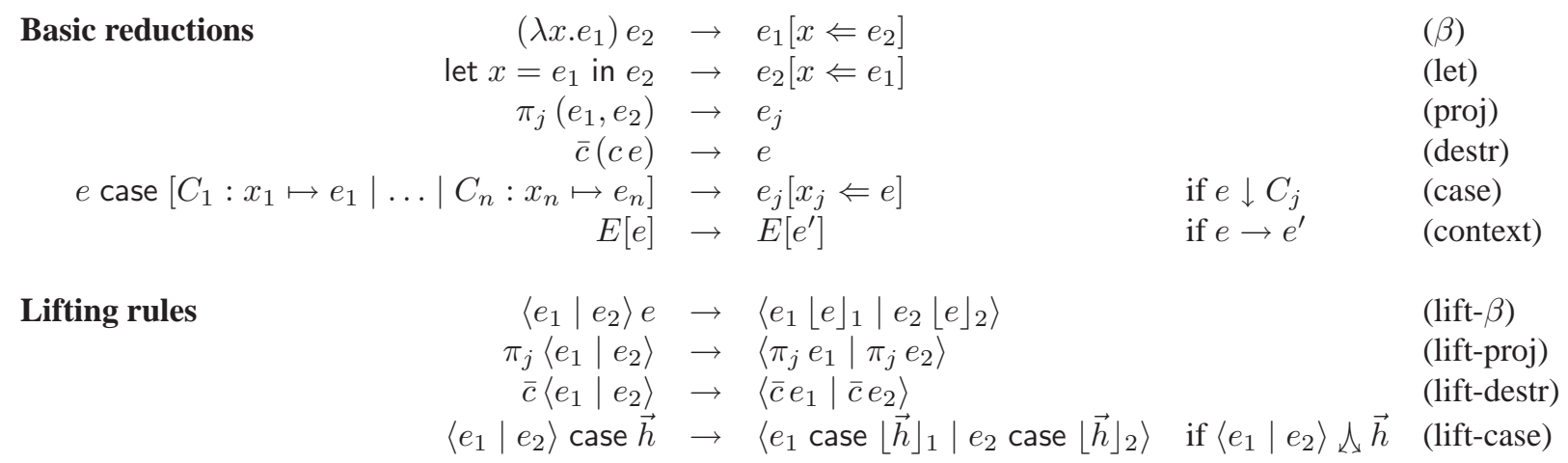

Figure 1. Semantics of $\lambda_{+}^{2}$

\subsection{Semantics}

The small step operational semantics of $\lambda_{+}^{2}$ is given in figure 1. The semantics of $\lambda_{+}$is obtained as a fragment of that of $\lambda_{+}^{2}$. To clarify the presentation, we divide the set of reduction rules into two groups. Basic reductions are those of $\lambda_{+}$. They perform computation. When read as $\lambda_{+}^{2}$ reduction rules, they may be applied outside brackets (in this case, the two projections perfom the same reduction step) or within a bracket context (then one of the two projections remains unchanged). Lifting rules are specific to $\lambda_{+}^{2}$ and deal with sharing. They have no computational: they leave both projections unchanged. Their purpose is only to prevent $\langle\cdot \mid \cdot\rangle$ constructs from blocking reduction by lifting them up (and thus duplicating some sub-terms).

We choose a call-by-name semantics (we will deal with call-by-value in section 4.6). Thus, evaluation contexts are defined as follows:

$$
\begin{aligned}
E::= & {[] e\left|\pi_{j}[]\right| \bar{c}[] \mid[] \text { case }[h|\ldots| h] } \\
& |\langle[] \mid e\rangle|\langle e \mid[]\rangle
\end{aligned}
$$

(The two last forms of context are specific to $\lambda_{+}^{2}$, allowing to apply basic reduction rules within a bracket construct.)

The rule (case) defines the semantics of case clauses. We write $e \downarrow C$ (read: e matches $C$ ) if and only if $e=c e^{\prime}$ for some $c \in C$ or $e=\left\langle c_{1} e_{1}^{\prime} \mid c_{2} e_{2}^{\prime}\right\rangle$ for some $c_{1} \in C$ and $c_{2} \in C$. For instance, by application of the (case) rule, the expression $c e^{\prime}$ case $\left[C_{1}: x_{1} \mapsto e_{1}|\ldots| C_{n}: x_{n} \mapsto e_{n}\right]$ reduces to $e_{j}\left[x_{j} \Leftarrow c e^{\prime}\right]$ if $c \in C_{j}$. Our semantics of case keeps good track of sharing since values like $\left\langle c_{1} e_{1}^{\prime} \mid c_{2} e_{2}^{\prime}\right\rangle$ may be matched without any lifting if the two head constructors fall within the same $C_{j}$. If they do not, (lift-case) must be applied before reducing the two resulting case subexpressions separately: $\left\langle e_{1} \mid e_{2}\right\rangle \downarrow\left[C_{1}: * \mapsto *|\ldots|\right.$ $\left.C_{n}: * \mapsto *\right]$ holds if and only if there exist distinct indices $j_{1}$ and $j_{2}$ such that $e_{1} \downarrow C_{j_{1}}$ and $e_{2} \downarrow C_{j_{2}}$.

\subsection{Relating $\lambda_{+}^{2}$ to $\lambda_{+}$}

An expression $e$ is a normal form if it is irreducible. We denote by the meta-variable $n$ any expression which is a normal form. The following lemma relates $\lambda_{+}^{2}$ normal forms to those of $\lambda_{+}$.

Lemma 3.1 (Normal forms) If $e$ is a normal form then $\lfloor e\rfloor_{1}$ or $\lfloor e\rfloor_{2}$ is a normal form.

Proof. By induction on the structure of $e$.

$\circ$ Cases $e=k, e=x, e=\lambda x \cdot e^{\prime}, e=c e .\lfloor e\rfloor_{1}$ and $\lfloor e\rfloor_{2}$ are both normal forms.

$\circ$ Case $e=e_{1} e_{2}$. Because (context) is not applicable, $e_{1}$ is a normal form. By induction hypothesis, $\left\lfloor e_{1}\right\rfloor_{i}$ is a normal form for some $i \in\{1,2\}$. Because neither $(\beta)$ nor (lift- $\beta$ ) is applicable, $e_{1}$ cannot be of the form $\langle * \mid *\rangle$ or $\lambda *$.*. It follows that $\left\lfloor e_{1}\right\rfloor_{i}$ is not a $\lambda$-abstraction. We conclude that $\lfloor e\rfloor_{i}$ is a normal form.

$\circ$ Cases $e=\pi_{j} e^{\prime}, e=\bar{c} e^{\prime}$. Similar to the previous case.

$\circ$ Case $e=$ let $x=e_{1}$ in $e_{2}$. $e$ is not a normal form.

- Case $e=e^{\prime}$ case $\vec{h}$. Because neither (case) nor

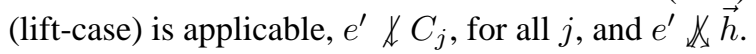

If $e^{\prime}=\langle * \mid *\rangle$ it follows that $\left\lfloor e^{\prime}\right\rfloor_{i}$ is not of the form $c *$ with $c \in C_{1} \cup \ldots \cup C_{n}$ for some $i \in\{1,2\}$. Because (context) is not applicable, both $\left\lfloor e^{\prime}\right\rfloor_{1}$ and $\left\lfloor e^{\prime}\right\rfloor_{2}$ are normal forms. We conclude that $\lfloor e\rfloor_{i}$ is a normal form.

Otherwise, $\left\lfloor e^{\prime}\right\rfloor_{i}$ is not of the form $c *$ with $c \in C_{1} \cup \ldots \cup$ $C_{n}$ for all $i \in\{1,2\}$. Because (context) is not applicable, $e^{\prime}$ is a normal form. Then, by induction hypothesis, $\left\lfloor e^{\prime}\right\rfloor_{i}$ is a normal form for some $i \in\{1,2\}$. It follows that $\lfloor e\rfloor_{i}$ is a normal form.

$\circ$ Case e $=\left\langle e_{1} \mid e_{2}\right\rangle$. Because (context) is not applicable with $E=\langle[] \mid *\rangle$ or $\langle * \mid[]\rangle,\lfloor e\rfloor_{1}$ and $\lfloor e\rfloor_{2}$ are both normal forms. 
We now show that every $\lambda_{+}^{2}$ reduction correctly simulates the simultaneous reduction of two $\lambda_{+}$expressions. This is expressed by two lemmas of soundness and correctness. The former states that the projections of a $\lambda_{+}^{2}$ reduction are valid $\lambda_{+}$reductions. The latter ensures that if both projections of an expression can produce a normal form then so can the expression. The proof techniques are almost identical to those used in [13].

Lemma 3.2 (Soundness) Let $i \in\{1,2\}$. If $e \rightarrow e^{\prime}$, then $\lfloor e\rfloor_{i} \rightarrow=\left\lfloor e^{\prime}\right\rfloor_{i}$.

Proof. By inspection of the reduction rules.

Lemma 3.3 (Completeness) Assume $\lfloor e\rfloor_{i} \rightarrow^{\star} n_{i}$ for all $i \in\{1,2\}$. Then, there exists $n$ such that $e \rightarrow^{\star} n$.

Proof. Because each of the "lift" reduction rules moves some $\langle\cdot \mid \cdot\rangle$ constructor strictly closer to the term's root, no infinite reduction sequence can consist exclusively of instances of these rules. As a consequence, if $e$ admits an infinite reduction sequence, then lemma 3.2 yields an infinite one out of $\lfloor e\rfloor_{i}$ for some $i \in\{1,2\}$. However, this is impossible, because both $\lfloor e\rfloor_{1}$ and $\lfloor e\rfloor_{2}$ can be reduced to normal forms, and the semantics of the $\lambda_{+}$fragment is deterministic. We conclude that there exists a normal form $n$ such that $e \rightarrow^{\star} n$.

\section{Typing}

Given two distinct constructors $c_{1}$ and $c_{2}$, we denote by $c_{1} \cdot c_{2}$ the (unordered) pair $\left\{c_{1}, c_{2}\right\}$. For any $C \subseteq \mathcal{C}$, let $C^{2}$ denotes the set of such pairs of elements of $C$.

Is this section, we present a type system tracing information flow in $\lambda_{+}$. This is a ground type system, in so far as it has no type variables. It handles polymorphism in an extensional way: a type scheme is represented by the set of its ground instances. This presentation is very amenable to proofs. Nevertheless, it does not describe a type inference algorithm: we will address this issue in section 5.

\subsection{The type algebra}

Let $(\mathcal{L}, \leq)$ be a lattice whose elements, denoted by $\ell$, represent security levels. Types, alternatives, rows and matrices are respectively defined as follows:

$$
\begin{aligned}
t & ::=\text { int }^{\ell}|t \rightarrow t| t \times t \mid r^{q} \\
a & ::=\text { Abs } \mid \text { Pret } \\
r & ::=\{c \mapsto a\}_{c \in C} \\
q & ::=\left\{c_{1} \cdot c_{2} \mapsto \ell\right\}_{c_{1} \cdot c_{2} \in C^{2}}
\end{aligned}
$$

A row (resp. a matrix) is an infinite, almost constant family of alternatives (resp. levels) indexed by a subset of $\mathcal{C}$ (resp. $\mathcal{C}^{2}$ ), its domain. (A family is almost constant if all but a finite number of its entries are equal.) It is worth noting that because pairs of $\mathcal{C}^{2}$ are unordered, matrices are in fact triangular. We write $(c: a ; r)$ for the row whose element at index $c$ is $a$ and whose other elements are given by the sub-row $r$, which is indexed by $\mathcal{C} \backslash\{c\}$. We denote by $\partial_{C} a$ the row indexed by $C$ that maps all its entries to $a$ (the domain $C$ may be omitted when it can be deduced from the context). $r_{\mid C}$ represents the row of the same domain as $r$ which is equal to $r$ on $C$ and Abs elsewhere. Similarly, we write $\partial_{C}^{2} \ell$ for the matrix indexed by $C^{2}$ that maps all its entries to $\ell$ and $q_{\mid C}$ denotes the row of the same domain as $q$ which is equal to $q$ on $C^{2}$ and $\perp$ elsewhere. Lastly, $\left(r^{q}\right)_{\mid C}$ is a shorthand for $r_{1}{ }^{q_{1}}$ where $r_{1}=r_{\mid C}$ and $q_{1}=q_{\mid C}$. Given a matrix $q$ and a set of constructors $C$, we define $q(C)=\sqcup\left\{q\left(c \cdot c^{\prime}\right) \mid c \in C, c^{\prime} \notin C\right\}$.

Our types are those of ML's type system (with rows for sum types [14]) decorated with security annotations that are simple levels $\ell$ and matrices $q$.

The type int $^{\ell}$ describes integer expressions whose value may reflect information of security level $\ell$.

In many type systems tracing information flow $[8,3,12$, 21,13 , arrows carry a security level representing information about the function's identity. Nevertheless, because the only way to observe a function consists in applying it and examining its results, there is really no difference between a function whose identity is secret and a function that produces secret results. As a consequence, following [1], we do not equip the $\rightarrow$ type constructor with an external security level: all the security annotations related to a function may be carried by its result type. Similarly, all of the information carried by a tuple is in fact carried by its components. Thus products have no security annotations.

The main novelty resides in sum types, such as $r^{q}$, which consist of a row and a matrix. First, following [14], the row $r$ indicates for every constructor $c \in \mathcal{C}$ if the given expression may (Pret) or may not (Abs) produce a value whose head constructor is $c$. The constructor Pre carries, in addition, the type of the constructor's argument. Second, for every pair of constructors $c_{1} \cdot c_{2} \in \mathcal{C}^{2}, q\left(c_{1} \cdot c_{2}\right)$ gives an approximation of the level of information leaked by revealing that the expression produces a value whose head constructor is $c_{1}$ rather than $c_{2}$, or symmetrically $c_{2}$ rather than $c_{1}$. Note that if $r(c)=$ Abs, none of the levels $q(c \cdot *)$ carries in practice any relevant information. Thus, the type system would have the same expressiveness if we considered only sum types $r^{q}$ such that $r(c)=$ Abs implies $q(c \cdot *)=\perp$. Nevertheless, we prefer not to introduce such a constraint which may needlessly complicate the presentation.

As usual in type systems tracing information flow, we equip the algebra with a subtyping relation $\leq$ that ex- 


$$
\begin{array}{ccc}
\text { int }^{\oplus} \quad \ominus \rightarrow \oplus \quad \oplus \times \oplus \quad \oplus^{\oplus} \quad \text { Pre } \oplus \\
\text { Abs } \leq \text { Pre } * & \{* \mapsto \oplus\} & \{* \cdot * \mapsto \oplus\}
\end{array}
$$

Figure 2. Subtyping

tends the ordering over information levels. This allows giving a directed view of the program's information flow graph. It is defined by the axioms in figure $2 . \oplus$ and $\ominus$ stand respectively for covariant and contravariant arguments. For instance, the axiom $\oplus^{\oplus}$ is an abbreviation of $r_{1}{ }^{q_{1}} \leq r_{2}{ }^{q_{2}} \Leftrightarrow r_{1} \leq r_{2} \wedge q_{1} \leq q_{2}$. Similarly, the last two axioms extend $\leq$ pointwise on rows and matrices, respectively.

\subsection{Guards}

We introduce a two-place predicate $\left[\ell_{1}, \ldots, \ell_{n}\right] \Downarrow$ $\left[t_{1}, \ldots, t_{n}\right]$ whose first argument is a (finite) list of security levels and whose second argument is a list of types of the same length. We also write $\left[t_{1}, \ldots, t_{n}\right] \leq t$ as a shorthand for $t_{1} \leq t \wedge \ldots \wedge t_{n} \leq t$ and $\ell \leqslant\left[t_{1}, \ldots, t_{n}\right]$ for $[\ell, \ldots, \ell] \preccurlyeq\left[t_{1}, \ldots, t_{n}\right]$.

In practice, we will use constraints of the form $\left[\ell_{1}, \ldots, \ell_{n}\right] \leqslant\left[t_{1}, \ldots, t_{n}\right] \leq t$ to record potential information flow at a point of the program where the execution path may take one of $n$ possible branches, depending on the result of a series of tests. The security level $\ell_{j}$ is intended to describe the information revealed by the test which guards the $j^{\text {th }}$ branch, and $t_{j}$ is the type of that branch's result. Last, $t$ is the type of the whole expression. It must be at least the union of all $t_{j}$ (i.e. $\left[t_{1}, \ldots, t_{n}\right] \leq t$ ) but it must also keep track of the information which the series of tests may leak. (Although the set of types is not a lattice, we define the union of a finite list of types $t_{1}, \ldots, t_{n}$ as the smallest type $t$ such that $t_{1} \leq t \wedge \ldots \wedge t_{n} \leq t$ if there exists one.)

We now comment the rules of figure 3 defining the predicate $\left[\ell_{1}, \ldots, \ell_{n}\right] \triangleq\left[t_{1}, \ldots, t_{n}\right]$. Because we intend to compute the union $t$ of $t_{1}, \ldots, t_{n}$, $\Vdash$ first constrains them to have the same structure. If $t_{1}, \ldots, t_{n}$ are integer types int $^{\ell_{1}^{\prime}}, \ldots$, int $^{\ell_{n}^{\prime}}$, GUARDS-INT requires each integer type $t_{j}$ to have security level $\ell_{j}$ or greater. Consequently, that of $t$ will be constrained to be $\ell_{1} \sqcup \ldots \sqcup \ell_{n}$ or greater and will record all potential information flows. Because $\rightarrow$ and $\times$ types carry no security annotation, rules GUARDS-FUN and GUARDS-PAIR propagate down the constraint on the result's type for $\rightarrow$ and the components types for $\times$. This reflects the fact, as explained in section 4.1, that all information about the identity of a function is given by its results and all information carried by a tuple is carried by its components. Lastly, GUARDS-SUM handles sum types.
Its first premise propagates the constraint down. The second one constrains the matrix. If two different branches $j_{1}$ and $j_{2}$ may produce results with different head constructors, namely $c_{1}$ and $c_{2}$, then any further test that distinguishes these head constructors is liable to leak information of level $\ell_{j_{1}} \sqcup \ell_{j_{2}}$. As a result, we constrain the field $c_{1} \cdot c_{2}$ in $q_{j_{1}}$ (resp. $q_{j_{2}}$ ) to be $\ell_{j_{1}}$ (resp. $\ell_{j_{2}}$ ) or greater. Thus, the same field in $t$ must be greater than or equal to $\ell_{j_{1}} \sqcup \ell_{j_{2}}$.

Our guards allow keeping more precise information about flows than the simple guards of [13]. To illustrate this point, let us consider the following example:

$$
\text { let } \begin{aligned}
t=x \text { case }[ & \{A\}: y \mapsto \text { if } \bar{A} y \text { then } A_{1} \text { else } A_{2} \\
& \{B\}: y \mapsto \text { if } \bar{B} y \text { then } B_{1} \text { else } B_{2} \\
& \left.\{D\}: y \mapsto \text { if } \bar{D} y \text { then } D_{1} \text { else } D_{2}\right]
\end{aligned}
$$

Because the guard constraint can consider the type of each branch of the case construct in isolation rather than only their union, the type system will be able to take into account the fact that, in this example, only the first (resp. second, third) branch can produce $A_{1}$ or $A_{2}$ (resp. $B_{1}$ or $B_{2}, D_{1}$ or $D_{2}$ ). As a result, the levels associated with the pairs $A_{1} \cdot A_{2}$, $B_{1} \cdot B_{2}$ and $D_{1} \cdot D_{2}$ are not constrained to be greater than the security levels attached to the identifier $x$ (i.e. $q(A$. $B), q(A \cdot D)$ and $q(B \cdot D)$ if $x$ has type $*^{q}$.) Using the intermediate result $t$, we now compute an integer $u$ :

$$
\text { let } \begin{aligned}
u=t \text { case }\left[\left\{A_{1}\right\}::_{-} \mapsto 0\right. \\
\left.\mathcal{C} \backslash\left\{A_{1}\right\}::_{-} \mapsto 1\right]
\end{aligned}
$$

Therefore the security level of the integer $u$ will in particular not be constrained to be greater than $q(B \cdot D)$, reflecting the fact that $u$ 's value carries no information about whether $t$ 's head constructor is $B$ rather than $D$.

Lemma 4.1 (Subset) If $\left[\ell_{1}, \ldots, \ell_{n}\right] \leqslant\left[t_{1}, \ldots, t_{n}\right]$ then for all $\left\{i_{1}, \ldots, i_{m}\right\} \subseteq\{1, \ldots, n\},\left[\ell_{i_{1}}, \ldots, \ell_{i_{m}}\right] \triangleq$ $\left[t_{i_{1}}, \ldots, t_{i_{m}}\right]$ holds.

Lemma 4.2 (Transitivity) If $\ell_{1}^{\prime} \leq \ell_{1}, \ldots, \ell_{n}^{\prime} \leq \ell_{n}$ and $\left[\ell_{1}, \ldots, \ell_{n}\right] \Vdash\left[t_{1}, \ldots, t_{n}\right]$ then $\left[\ell_{1}^{\prime}, \ldots, \ell_{n}^{\prime}\right] \leqslant\left[t_{1}, \ldots, t_{n}\right]$ holds.

Proof. By induction on the derivation of $\left[\ell_{1}, \ldots, \ell_{n}\right] \lessgtr$ $\left[t_{1}, \ldots, t_{n}\right]$.

\subsection{Additional notations}

A polytype $s$ is a nonempty set of types. A polytype environment $\Gamma$ is a partial mapping from program variables to polytypes. $\Gamma[x \mapsto s]$ denotes the environment which maps $x$ to $s$ and agrees with $\Gamma$ otherwise. A type judgement $\Gamma \vdash e: t$ is a triple of a polytype environment, an expression and a type. (We also write $\Gamma \vdash e: s$ when $\Gamma \vdash e: t$ holds for all $t \in s$.) 
Types

$$
\begin{aligned}
& \text { GUARDS-INT } \\
& \frac{\ell_{1} \leq \ell_{1}^{\prime} \cdots \ell_{n} \leq \ell_{n}^{\prime}}{\left[\ell_{1}, \ldots, \ell_{n}\right] \lessgtr\left[\operatorname{int}^{\ell^{\prime}}, \ldots, \text { int }^{\ell_{n}^{\prime}}\right]} \\
& \text { GUARDS-FUN } \\
& \text { GUARDS-SUM } \\
& \frac{\left[\ell_{1}, \ldots, \ell_{n}\right] \Vdash\left[t_{1}, \ldots, t_{n}\right]}{\left[\ell_{1}, \ldots, \ell_{n}\right] \Vdash\left[t_{1}^{\prime} \rightarrow t_{1}, \ldots, t_{n}^{\prime} \rightarrow t_{n}\right]} \\
& \text { GUARDS-PAIR } \\
& \begin{array}{c}
{\left[\ell_{1}, \ldots, \ell_{n}\right] \Vdash\left[t_{1}, \ldots, t_{n}\right]} \\
\frac{\left[\ell_{1}, \ldots, \ell_{n}\right] \Vdash\left[t_{1}^{\prime}, \ldots, t_{n}^{\prime}\right]}{\left[\ell_{1}, \ldots, \ell_{n}\right] \Vdash\left[t_{1} \times t_{1}^{\prime}, \ldots, t_{n} \times t_{n}^{\prime}\right]}
\end{array} \\
& \frac{\forall j_{1} \neq j_{2}, c_{1} \neq c_{2}, r_{j_{1}}\left(c_{1}\right) \neq \operatorname{Abs} \wedge r_{j_{2}}\left(c_{2}\right) \neq \operatorname{Abs} \Rightarrow \ell_{j_{1}} \leq q_{j_{1}}\left(c_{1} \cdot c_{2}\right) \wedge \ell_{j_{2}} \leq q_{j_{2}}\left(c_{1} \cdot c_{2}\right)}{\left[\ell_{1}, \ldots, \ell_{n}\right] \Vdash\left[r_{1}^{q_{1}}, \ldots r_{n}^{q_{n}}\right]}
\end{aligned}
$$

Rows

$$
\begin{aligned}
& \text { GUARDS-ALT } \\
& \frac{\left[\ell_{i_{1}}, \ldots, \ell_{i_{m}}\right] \Vdash\left[t_{i_{1}}, \ldots, t_{i_{m}}\right]}{\left[\ell_{1}, \ldots, \ell_{n}\right] \Vdash\left[\mathrm{Abs}^{\star}, \operatorname{Pre} t_{i_{1}}, \text { Abs }^{\star}, \ldots, \text { Abs }^{\star}, \text { Pre }_{i_{m}}, \text { Abs }^{\star}\right]}
\end{aligned}
$$$$
\begin{aligned}
& \text { GUARDS-ROW } \\
& \frac{\forall c \in \mathcal{C},\left[\ell_{1}, \ldots, \ell_{n}\right] \Vdash\left[r_{1}(c), \ldots, r_{n}(c)\right]}{\left[\ell_{1}, \ldots, \ell_{n}\right] \star\left[r_{1}, \ldots, r_{n}\right]}
\end{aligned}
$$

Figure 3. Guards

Given a row $r$ and a set of constructors $C$, we say that $r$ cuts $C(r \cap C)$ if and only if there exists $c \in C$ and a type $t$ such that $r(c)=$ Pre $t$. Similarly, $r$ is included in $C$ $(r \Subset C)$ if and only if every $c$ such that $r(c)=$ Pre $*$ is in C.

\subsection{Typing rules}

Because the security lattice $(\mathcal{L}, \leq)$ is arbitrary, our proof technique requires to temporarily split security levels between low and high ones. That's the reason why, in the present section and the next one, we assume fixed $H$, an upward-closed subset of $\mathcal{L}$ whose elements will be considered as high security levels. Full generality will be recovered in section 4.6.

$\lambda_{+}$'s typing rules are given in figure 4. INT assigns a base type to integer constants, with an unconstrained security level. Because security annotations appear only on sum nodes and leaves in types, rules VAR, ABS, APP, LET, PAIR and PROJ involve no particular constraint and are identical to those of [11]. Polymorphism is allowed by rule LET: $e_{1}$ can be given a polytype $s$. Therefore, by VAR, each occurrence of $x$ within $e_{2}$ can be typed with a different $t \in s$. Rule E-APP of [13] differs from APP (regardless of the annotations concerning side-effects) by an extra constraint $\ell \triangleleft t$, where $\ell$ is the security annotation of the $\rightarrow$ type constructor. Because the function's result may reveal information about the identity of the function itself, its type $t$ must be guarded by $\ell$. In this paper, potential information about the function's identity is directly propagated to its result type by $₫$. As a result, no extra constraint is needed in the premises of rule APP.

INJ associates to the expression $c e$ a row which maps $c$ to Pret (where $t$ is $e$ 's type) and leaves other entries unconstrained, allowing them to be Abs. The matrix is alike unconstrained (and may be $\partial \perp$ ) since knowing the head constructor of the value cannot reveal any information at this point. DESTR requires $\bar{c}$ 's argument to have type $(c \text { : Pre } * ; \partial \mathrm{Abs})^{*}$. This statically ensures that its headconstructor is $c$, allowing the matrix to be ignored. It would in particular be the case for a value matched by $\{c\}$ in a case expression.

Let us now consider rule CASE. Its first premise simply specifies the type of the matched expression, which is expected to be a sum type. The condition $r \Subset C_{1} \cup \ldots \cup C_{n}$ ensures that the matching is exhaustive: a handler must be provided for each head constructor that $e$ may exhibit. The third premise concerns handlers. The handler $e_{j}$ is considered only if it is liable to be invoked (i.e. $r \cap C_{j}$ ). It is typechecked in an environment where the type assigned to the program variable $x_{j}$ is nothing but the "restriction" to $C_{j}$ of the matched expression's type. The type given to $x_{j}$ is therefore more precise than that of $e$, reflecting the success of the test guarding the handler. A security level $q\left(C_{j}\right)=\sqcup\left\{q\left(c \cdot c^{\prime}\right) \mid c \in C_{j}, c^{\prime} \notin C_{j}\right\}$ is associated to each handler. It is an approximation of the information leaked by revealing whether the $e$ 's head constructor belongs to $C_{j}$ (i.e. whether $e_{j}$ will be executed). Then, the last premise computes the union $t$ of all $t_{j}$ guarded by these levels as described in section 4.2.

Rule BRACKET is specific to $\lambda_{+}^{2}$. It allows typing $\langle\cdot \mid \cdot\rangle$ constructs by computing the union $t$ of the types of the two sub-expressions. Moreover, because brackets enclose secret parts of a computation, they must receive high type, i.e. $t$ must be guarded by arbitrary levels chosen in $H$. 


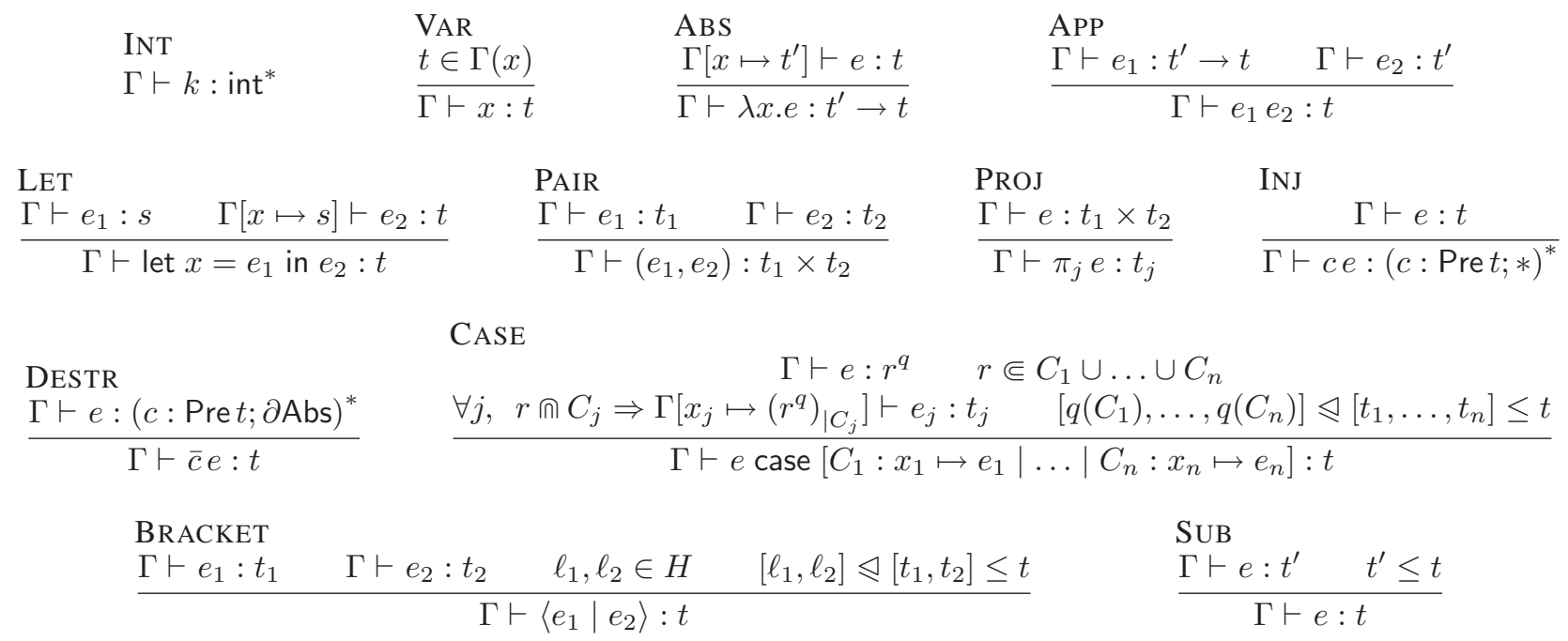

\section{Figure 4. The type system for $\lambda_{+}^{2}$}

\subsection{Type soundness}

We first state a few auxiliary lemmas whose proofs are straightforward, then establish the subject reduction theorem.

Lemma 4.3 (Projection) Let $i \in\{1,2\}$. If $\Gamma \vdash e: t$ then $\Gamma \vdash\lfloor e\rfloor_{i}: t$.

Lemma 4.4 (Guard) If $\Gamma \vdash\left\langle e_{1} \mid e_{2}\right\rangle: t$ then there exist $\ell_{1}, \ell_{2} \in H$ and two types $t_{1}, t_{2}$ such that $\Gamma \vdash e_{1}: t_{1}$, $\Gamma \vdash e_{2}: t_{2}$ and $\left[\ell_{1}, \ell_{2}\right] \Vdash\left[t_{1}, t_{2}\right] \leq t$.

Lemma 4.5 (Sum) Assume $\Gamma \vdash e: r^{q}$. If $e \downarrow C$ then $\Gamma \vdash e:\left(r^{q}\right)_{\mid C}$ and $r \cap C$.

Lemma 4.6 (Substitution) Assume $\vdash e^{\prime}: s$. Then $\Gamma[x \mapsto$ $s] \vdash e: t$ implies $\Gamma \vdash e\left[x \Leftarrow e^{\prime}\right]: t$.

Theorem 4.1 (Subject reduction) Let $e \rightarrow e^{\prime}$. If $\vdash e: t$ then $\vdash e^{\prime}: t$.

Proof. By induction on the derivation of $e \rightarrow e^{\prime}$. We assume, w.l.o.g., that the derivation of $\vdash e: t$ does not end with an instance of SUB. As a result, it must end with an instance of the single syntax-directed rule that matches $e$ 's structure.

$\circ$ Case $(\beta) . e$ is $\left(\lambda x . e_{1}\right) e_{2}$ and $e^{\prime}$ is $e_{1}\left[x \Leftarrow e_{2}\right]$. In ApP's premises, we have $\vdash \lambda x . e_{1}: t^{\prime} \rightarrow t$ and $\vdash e_{2}: t^{\prime}$. The former must end with an instance of ABS, followed by a number of instances of SUB. Because $\rightarrow$ is contravariant (resp. covariant) in its first (resp. second) parameter, applying SUB to ABS's premise yields $\left(x \mapsto t^{\prime \prime}\right) \vdash e_{2}: t$ for some $t^{\prime \prime}$ such that $t^{\prime} \leq t^{\prime \prime}$. By SuB, $\vdash e_{2}: t^{\prime \prime}$ holds. Then lemma 4.6 yields $\vdash e_{1}\left[x \Leftarrow e_{2}\right]: t$.

- Case (let). By LET and lemma 4.6.

- Case (proj). $e$ is $\pi_{j}\left(e_{1}, e_{2}\right)$ and $e^{\prime}$ is $e_{j}$. In PROJ's premises, we have $\vdash\left(e_{1}, e_{2}\right): t_{1} \times t_{2}$ with $t_{j}=t$. This derivation must end by an instance of PAIR followed by a number of instances of SUB. It follows that $\vdash e_{j}: t_{j}$, i.e. $\vdash e^{\prime}: t$.

- Case (destr). $e$ is $\bar{c}\left(c e^{\prime}\right)$. In Destr's premises, we have $\vdash c e^{\prime}:(c: \text { Pre } t ; *)^{*}$. By Sub and INJ, this yields $\vdash e^{\prime}: t$.

- Case (case). $e$ is $e_{0}$ case $\left[C_{1}: x_{1} \mapsto e_{1}|\ldots| C_{n}\right.$ : $\left.x_{n} \mapsto e_{n}\right]$ and $e^{\prime}$ is $e_{j}\left[x_{j} \Leftarrow e_{0}\right]$, with $e_{0} \downarrow C_{j}$. Within CASE's premises, we have $\vdash e_{0}: r^{q}$ and $\left(x_{j} \mapsto\left(r^{q}\right)_{\mid C_{j}}\right) \vdash$ $e_{j}: t_{j}$ with $t_{j} \leq t$. By lemma 4.5, the former yields $\vdash e_{0}$ : $\left(r^{q}\right)_{\mid C_{j}}$. Applying lemma 4.6 and SuB, we obtain $\vdash e^{\prime}: t$.

$\circ$ Case (lift- $\beta$ ). $e$ is $\left\langle e_{1} \mid e_{2}\right\rangle e_{0}$ and $e^{\prime}$ is $\left\langle e_{1}\left\lfloor e_{0}\right\rfloor_{1}\right|$ $\left.e_{2}\left\lfloor e_{0}\right\rfloor_{2}\right\rangle$. In ApP's premises, we have $\vdash\left\langle e_{1} \mid e_{2}\right\rangle: t^{\prime} \rightarrow t$ and $\vdash e_{0}: t^{\prime}$. Lemma 4.3 yields $\vdash\left\lfloor e_{0}\right\rfloor_{1}: t^{\prime}$ and $\vdash\left\lfloor e_{0}\right\rfloor_{2}$ : $t^{\prime}$. By lemma 4.4 and GuARDS-Fun, $\vdash e_{1}: t_{1}^{\prime} \rightarrow t_{1}$ and $\vdash e_{2}: t_{2}^{\prime} \rightarrow t_{2}$ hold for some $t_{1}, t_{2}, t_{1}^{\prime}, t_{2}^{\prime}$ such that $\left[\ell_{1}, \ell_{2}\right] \unlhd\left[t_{1}, t_{2}\right] \leq t$ (with $\left.\ell_{1}, \ell_{2} \in H\right), t_{1}^{\prime} \geq t^{\prime}$ and $t_{2}^{\prime} \geq t^{\prime}$. By SUB and APP, it follows $\vdash e_{1}\left\lfloor e_{0}\right\rfloor_{1}: t_{1}$ and $\vdash e_{2}\left\lfloor e_{0}\right\rfloor_{2}$ : $t_{2}$. By BRACKET, we conclude that $\vdash e^{\prime}: t$.

$\circ$ Case (lift-proj). $e$ is $\pi_{j}\left\langle e_{1} \mid e_{2}\right\rangle$ and $e^{\prime}$ is $\left\langle\pi_{j} e_{1}\right|$ $\left.\pi_{j} e_{2}\right\rangle$. In PROJ's premises, we find $\vdash\left\langle e_{1} \mid e_{2}\right\rangle: t_{1} \times t_{2}$ with $t_{j}=t$. By lemma 4.4 and GUARDS-PAIR, $\vdash e_{1}: t_{11} \times t_{12}$ and $\vdash e_{2}: t_{21} \times t_{22}$ hold for some $t_{11}, t_{12}, t_{21}, t_{22}$ with in particular $\left[\ell_{1}, \ell_{2}\right] \lessgtr\left[t_{1 j}, t_{2 j}\right] \leq t_{j}$ for some $\ell_{1}, \ell_{2} \in H$. By PROJ, it follows that $\vdash \pi_{j} e_{1}: t_{1 j}$ and $\vdash \pi_{j} e_{2}: t_{2 j}$. Conclude by BRACKET. 
- Case (lift-destr). $\quad e$ is $\bar{c}\left\langle e_{1} \mid e_{2}\right\rangle$ and $e^{\prime}$ is $\left\langle\bar{c} e_{1}\right.$ $\left.\bar{c} e_{2}\right\rangle$. In DESTR's premises, we have $\vdash\left\langle e_{1} \mid e_{2}\right\rangle$ : $(c: \text { Pre } t ; \partial \mathrm{Abs})^{*}$. By lemma 4.4, we have $\vdash e_{1}$ : $\left(c: \text { Pre } t_{1} ; \partial \mathrm{Abs}\right)^{*}$ and $\vdash e_{2}:\left(c: \text { Pre } t_{2} ; \partial \mathrm{Abs}\right)^{*}$ for some $t_{1}, t_{2}$ such that $\left[\ell_{1}, \ell_{2}\right] \unlhd\left[t_{1}, t_{2}\right] \leq t$ with $\ell_{1}, \ell_{2} \in H$. By DESTR, it follows that $\vdash \bar{c} e_{1}: t_{1}$ and $\vdash \bar{c} e_{2}: t_{2}$. Conclude by BRACKET.

$\circ$ Case (lift-case). $e$ is $\left\langle e_{1}^{\prime} \mid e_{2}^{\prime}\right\rangle$ case $\left[C_{j}: x_{j} \mapsto e_{j}\right]_{j}$ and $e^{\prime}$ is $\left\langle e_{1}^{\prime}\right.$ case $\left.\left[C_{j}: x_{j} \mapsto\left\lfloor e_{j}\right\rfloor_{1}\right]_{j}\right| e_{2}^{\prime}$ case $\left[C_{j}: x_{j} \mapsto\right.$ $\left.\left.\left\lfloor e_{j}\right\rfloor_{2}\right]_{j}\right\rangle$. Because $\left\langle e_{1}^{\prime} \mid e_{2}^{\prime}\right\rangle \downarrow\left[C_{1}|\ldots| C_{n}\right], e_{1}^{\prime} \downarrow C_{j_{1}}$ and $e_{2}^{\prime} \downarrow C_{j_{2}}$ hold for some $j_{1} \neq j_{2}$.

In CASE's premises, we have $\vdash\left\langle e_{1}^{\prime} \mid e_{2}^{\prime}\right\rangle: r^{q}$ and $r \Subset$ $C_{1} \cup \ldots C_{n}$ and $r \cap C_{j_{i}} \Rightarrow\left(x \mapsto\left(r^{q}\right)_{\mid C_{j_{i}}}\right) \vdash e_{j_{i}}: t_{j_{i}}$ (for all $i \in\{1,2\})$ and $\left[q\left(C_{1}\right), \ldots, q\left(C_{n}\right)\right] \leqslant\left[t_{1}, \ldots, t_{n}\right] \leq t$.

By lemma 4.4, there exist $r_{1}, q_{1}, r_{2}$ and $q_{2}$ such that $\vdash e_{1}^{\prime}: r_{1}^{q_{1}}$ and $\vdash e_{2}^{\prime}: r_{2}{ }^{q_{2}}$ with $\left[\ell_{1}, \ell_{2}\right] \triangleq\left[r_{1}{ }^{q_{1}}, r_{2}{ }^{q_{2}}\right] \leq r^{q}$ for some $\ell_{1}, \ell_{2} \in H$. By lemma 4.5, we obtain $\vdash e_{i}^{\prime}$ : $\left(r_{i}{ }^{q_{i}}\right)_{\mid C_{j_{i}}}$ and $r_{i} \cap C_{j_{i}}$ (for all $i \in\{1,2\}$ ). The latter and $r_{j_{i}} \leq r$ imply $r$ ก $C_{j_{i}}$. Then $\left(x \mapsto\left(r^{q}\right)_{\mid C_{j_{i}}}\right) \vdash e_{j_{i}}$ : $t_{j_{i}}$ holds and, by lemma 4.3, this yields $\left(x \mapsto\left(r^{q}\right)_{\mid C_{j_{i}}}\right) \vdash$ $\left\lfloor e_{j_{i}}\right\rfloor_{i}: t_{j_{i}}$. Applying an instance of CASE, we obtain $\vdash$ $e_{i}^{\prime}$ case $\left[C_{j}: x_{j} \mapsto\left\lfloor e_{j}\right\rfloor_{i}\right]_{j}: t_{j_{i}}$.

$r_{1} \cap C_{j_{1}}, r_{2} \cap C_{j_{2}}$ and GUARDS-SUM imply $\ell_{1} \leq q_{1}\left(c_{1}\right.$. $\left.c_{2}\right)$ and $\ell_{2} \leq q_{2}\left(c_{1} \cdot c_{2}\right)$. It follows $\ell_{1} \leq q\left(C_{1}\right)$ and $\ell_{2} \leq$ $q\left(C_{2}\right)$. Then, because $\left[q\left(C_{1}\right), \ldots, q\left(C_{n}\right)\right] \leqslant\left[t_{1}, \ldots, t_{n}\right] \leq$ $t$, we have by lemmas 4.1 and $4.2,\left[\ell_{1}, \ell_{2}\right] \triangleq\left[t_{i_{1}}, t_{i_{2}}\right] \leq t$. Conclude by BRACKET.

$\circ$ Case (context). By induction hypothesis.

\subsection{Non-interference}

In the following, the set $H$ is no longer fixed. Thus, it appears as an extra parameter on $\lambda_{+}^{2}$ typing judgements (we write $\vdash_{H}$ instead of $\vdash$ ). It is still unnecessary to mention it on those judgements which involve $\lambda_{+}$expressions because $H$ is used only in the BRACKET rule.

Theorem 4.2 (Non-interference) Choose $\ell, h \in \mathcal{L}$ such that $h \not \leq \ell$. Let $h \lessgtr\left[t_{1}, t_{2}\right] \leq t$. Assume $(x \mapsto t) \vdash e$ : int ${ }^{\ell}$, where $e$ is a $\lambda_{+}$expression. If $\vdash e_{i}: t_{i}$ and $e\left[x \Leftarrow e_{i}\right] \rightarrow^{\star}$ $k_{i}$, for $i \in\{1,2\}$, then $k_{1}=k_{2}$.

Proof. Let $H$ be the upper cone $\left\{h^{\prime} \mid h \leq h^{\prime}\right\}$. Define $e^{\prime}=$ $\left\langle e_{1} \mid e_{2}\right\rangle$. By BRACKET, $\vdash_{H} e^{\prime}: t$. Lemma 4.6 yields $\vdash_{H}$ $e\left[x \Leftarrow e^{\prime}\right]$ : int ${ }^{\ell}$. Now, $\left\lfloor e\left[x \Leftarrow e^{\prime}\right]\right\rfloor_{i}$ is $e\left[x \Leftarrow e_{i}\right]$, which, by hypothesis, reduces to $k_{i}$. According to lemma 3.3, there exists a normal form $n$ such that $e\left[x \Leftarrow e^{\prime}\right] \rightarrow^{\star} n$. By theorem 4.1, $\vdash_{H} e\left[x \Leftarrow e^{\prime}\right]$ : int ${ }^{\ell}$ implies $\vdash_{H} n:$ int $^{\ell}$.

By lemma 3.1, $\lfloor n\rfloor_{i}$ is a normal form for some $i \in$ $\{1,2\}$. Because the semantics of the $\lambda_{+}$fragment is deterministic, $\lfloor n\rfloor_{i}=k_{i}$ must hold. It follows that $n$ is of the form $k$ or $\langle * \mid *\rangle$. If the latter, then by lemma 4.4, there exists $\ell^{\prime} \in H$ such that $\ell^{\prime} \leq \ell$, which implies $\ell \in H$, a contradiction. Thus, we must have $n=k=k_{i}$.

By lemma 3.2, $e_{1} \rightarrow^{\star} k$ and $e_{2} \rightarrow^{\star} k$ hold. It follows that $n_{1}=n_{2}=k$.

This theorem establishes a weak non-interference result in so far as it requires both expressions to converge. Indeed, in order to provide a fine-grained analysis, our type system is able to ignore some test conditions. For instance, define $e$ as $e^{\prime}$ case $\left[A:_{-} \mapsto D \mid B:_{-} \mapsto D\right]$, where $e^{\prime}$ is an arbitrary expression of type $(A, B$ : Pre $* ; \partial \mathrm{Abs})$. The type system statically detects that the result of $e$ 's evaluation does not depend on $e^{\prime}$. Yet $e$ 's termination does depend on that of $e^{\prime}$ : reducing the case clause requires $e^{\prime}$ to produce either $A$ or $B$ (even though it does not affect the final result). Obtaining a strong non-interference statement would require dropping the fine-grained treatment of sums. Anyway, it would be of little sense since we do not deal with timing leaks in general.

Because the type system satisfies a progress property (i.e. "no well-typed expression is stuck"), each hypothesis " $e\left[x \Leftarrow e_{i}\right]$ yields an integer" of the non-interference theorem can be safely weakened into " $e\left[x \Leftarrow e_{i}\right]$ does not diverge", i.e. $e\left[x \Leftarrow e_{i}\right] \rightarrow^{\star} n_{i}$, because, by progress, any $\lambda_{+}$ normal form of type int ${ }^{*}$ must be an integer constant.

The non-interference result still applies for $\lambda_{+}^{\mathrm{CBV}}$, the $\lambda_{+}{ }^{-}$ calculus equipped with a call-by-value semantics $\rightarrow_{\mathrm{CBV}}$ (we omit its definition because it is standard). Indeed, if $e\left[x \Leftarrow e_{1}\right] \rightarrow_{\mathrm{CBV}}^{*} k_{1}$ and $e\left[x \Leftarrow e_{1}\right] \rightarrow_{\mathrm{CBV}}^{*} k_{2}$ then, by normalization, $e\left[x \Leftarrow e_{1}\right] \rightarrow^{*} k_{1}$ and $e\left[x \Leftarrow e_{1}\right] \rightarrow^{*} k_{2}$. Applying theorem 4.2 with the correct hypothesis about types, we obtain $k_{1}=k_{2}$.

\section{Type inference}

We now explain how a type system with decidable type inference can be obtained from that of section 4 . This raises several technical issues. By lack of space we prefer to present it in an informal manner only.

The description of an inference algorithm for a constraint based type system generally consists of two distinct parts: a set of inference rules and a constraint solving algorithm. Obtaining inference rules in the style of $\mathrm{HM}(\mathrm{X})$ [17] from a set of rules such as that of figure 4 is a well-studied issue; the reader is referred to $[7,11]$ for more details. It requires introducing type variables, a constraint language and universally quantified, constrained type schemes. The correctness of the system thus obtained may be proven by a simple encoding of its judgements into $\lambda_{+}$judgements. This set of rules may be viewed as an algorithm which, given an input expression, returns a constraint which is satisfiable if and only if the expression is well typed.

Constraint solving for (non-atomic) subtyping is known to be decidable and reasonably efficient algorithms have 
been proposed in this area $[9,10]$. However, our system involves non-standard forms of constraints. We claim that constraint solving remains a decidable problem.

Constraints of the form $r \Subset C$ can be encoded using simple subtyping constraints requiring fields not in $C$ to be Abs. In CASE's premises, typing judgements concerning the handlers are subject to a condition of the form $r$ ก $C$. Such a condition may be enforced in the type inference system by prefixing with it every constraint produced by the judgement. This introduces conditional constraints such as $(r \cap C) ? \gamma$ where $\gamma$ is an arbitrary constraint. Such a constraint may be solved by keeping it unchanged as long as none of $r$ 's fields corresponding to constructors in $C$ are known to be Pre $*$. When one of them is unified with Pre* then the condition is satisfied and the conditional $(r \cap C) ? \gamma$ must be replaced by $\gamma$ itself.

Guards $\left[\ell_{1}, \ldots, \ell_{n}\right] \leqslant\left[t_{1}, \ldots, t_{n}\right] \leq t$ can be solved in a "lazy" manner. As long as nothing is known about the structure of $t$ (or any of the $t_{i}$ ), the constraint is preserved intact. When $t$ (or one of the $t_{i}$ ) is instantiated with a term, the constraint may be propagated throughout its structure to the leaves, generating a number of subconstraints. This propagation is straightforward on $\rightarrow$ and $\times$ nodes. At sum nodes, it requires the introduction of a form of conditional constraint: $\left[\ell_{1}, \ldots, \ell_{n}\right] \Vdash\left[r_{1} q_{1}, \ldots, r_{n}{ }^{q_{n}}\right] \leq r^{q}$ is indeed equivalent to $\left[\ell_{1}, \ldots, \ell_{n}\right] \preccurlyeq\left[r_{1}, \ldots, r_{n}\right] \leq r$ and $\forall c_{1} \neq$ $c_{2}, \quad(\operatorname{Pre} *, \operatorname{Pre} *) \leq\left(r_{j_{1}} \star r_{j_{2}}\right)\left(c_{1} \cdot c_{2}\right) \Rightarrow \ell_{j_{1}} \leq q_{j_{1}}\left(c_{1} \cdot c_{2}\right)$ for every $j_{1} \neq j_{2}\left(r_{j_{1}} \star r_{j_{2}}\right.$ denotes the matrix formed by the cartesian product of the rows $r_{j_{1}}$ and $r_{j_{2}}$ ).

If the number of constructor names that are present in every sum type is finite and statically known (e.g. if sum type are used to represent finite variant types such as ML's datatypes), such a constraint may be decomposed pointwise by generating a different conditional constraint for each pair of constructors $c_{1} \cdot c_{2}$. Otherwise, it may be viewed as a conditional constraint involving two-dimensional rows: (Pre $*$, Pre $*) \leq\left(r_{j_{1}} \star r_{j_{2}}\right) \Rightarrow \ell_{j_{1}} \leq q_{j_{1}}$. Although twodimensional rows have never been - to the best of our knowledge - really used, they form a natural generalization of rows [15] and can be manipulated using the same techniques [10].

Lastly, constraints of the form $q(C) \leq \ell$ are equivalent to $\forall c_{1} \in C, \forall c_{2} \in \mathcal{C} \backslash C, q\left(c_{1} \cdot c_{2}\right) \leq \ell$. Once again, if the number of involved constructor names is finite, this constraint may be decomposed pointwise into a number of inequalities between security levels. Otherwise, it may be viewed as a subtyping constraint between twodimensional rows: $q^{\prime} \leq \partial^{2} \ell$ (we denote by $\partial^{2} \ell$ the constant two-dimensional row with the same domain as $q^{\prime}$ ) where $q^{\prime}$ is the restriction of $q$ to the rectangle $C \times(\mathcal{C} \backslash C)$. Assuming $C$ is finite or cofinite, this restriction may be computed thanks to unification constraints using Rémy's row syntax [14].

\section{Examples}

In this section, we illustrate the expressiveness of $\lambda_{+}$'s type system by describing the types obtained for a small set of relevant examples. We use a Caml-like syntax, which can be easily de-sugared into $\lambda_{+}$. In particular, we allow constructors with no argument and booleans. Booleans can be easily encoded within sums by choosing two different constructors $T$ and $F$ (for the constants true and false, respectively). The test if $e_{1}$ then $e_{2}$ else $e_{3}$ can be translated into $e_{1}$ case $\left[\{T\}: e_{2} \mid\{F\}: e_{3}\right]$. Because it involves only two constructors, the type of a boolean expression $(T: \text { Pre } ; F: \text { Pre } ; \partial A b s)^{(T \cdot F: \ell ; *)}$ carries only one relevant security level, $\ell$. Thus, it may be abbreviated into $b^{\ell}$.

Our first examples are those of the introduction.

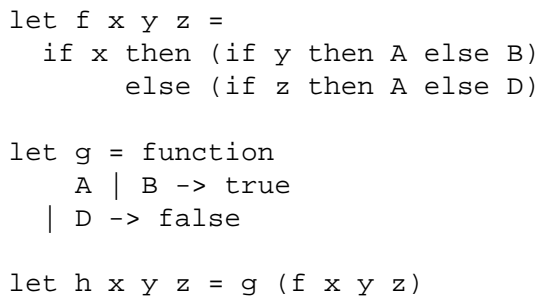

The function $f$ admits all types of the form $\mathrm{b}^{\alpha} \rightarrow \mathrm{b}^{\beta} \rightarrow \mathrm{b}^{\delta} \rightarrow$ $(A, B, D: \text { Pre } ; *)^{(A \cdot B: \alpha \sqcup \beta ; A \cdot D: \alpha \sqcup \delta ; B \cdot D: \alpha ; *)}$ (where $\alpha, \beta$ and $\delta$ are security levels). The tails of the row and the matrix describing the function's results are unconstrained, allowing them to be $\partial A$ bs and $\partial \perp$, respectively. As explained in the introduction, testing whether the result of $f$ is $B$ rather than $D$ can leak information only about the first argument. Therefore, the field $B \cdot D$ of the matrix is only constrained by the level $\alpha$. We obtain $(A, B, D: \text { Pre } ; \partial A \text { Abs })^{(A \cdot D, B \cdot D: \alpha ; *)} \rightarrow \mathrm{b}^{\alpha}$ for $g$. Lastly our system detects the absence of information flow from the second argument to the output of $h$, the composition of $f$ and $g$, as reflected by its types: $\mathrm{b}^{\alpha} \rightarrow \mathrm{b}^{\beta} \rightarrow \mathrm{b}^{\delta} \rightarrow \mathrm{b}^{\alpha \sqcup \delta}$.

The function $f^{\prime}$ is identical to the function $f$ but it returns its result embedded in a $\lambda$-abstraction:

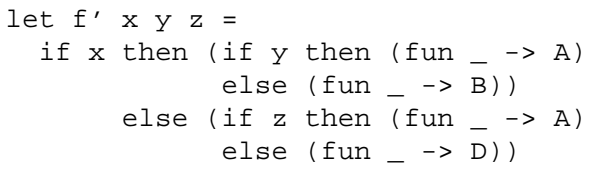

For $f^{\prime}, \quad$ the system gives $\mathbf{b}^{\alpha} \rightarrow \mathbf{b}^{\beta} \rightarrow \mathbf{b}^{\delta} \rightarrow(* \rightarrow(A, B, D$ : Pre;*) $(A \cdot B: \alpha \sqcup \beta ; A \cdot D: \alpha \sqcup \delta ; B \cdot D ; \alpha ; *)$. This example illustrates the interest of the absence of security level on the $\rightarrow$ type constructor: it allows the accuracy of the typing of sums to pass through it. As a result, if we re-implement $f$ by let $f x y z=\left(f^{\prime} x\right.$ y $\left.z\right)$, we obtain exactly the same type scheme as that for the first version.

Our next example tests whether the head constructor of its argument is $A$ or not: 


\section{Basic reductions}

$$
\begin{array}{rll}
(\lambda x . e) v & \rightarrow e[x \leftarrow v] & (\beta) \\
\pi_{j}\left(v_{1}, v_{2}\right) & \rightarrow v_{j} & \text { (proj) } \\
\text { let } x=v \text { in } e & \rightarrow e[x \leftarrow v] & \text { (let) }
\end{array}
$$

\section{Sequencing}

$$
\begin{aligned}
& \text { bind } x=v \text { in } e \rightarrow e[x \leftarrow v] \quad \text { (bind) } \\
& \text { raise }(\varepsilon v) \text { handle } \varepsilon x \succ e \rightarrow e[x \leftarrow v] \quad \text { (handle) } \\
& \text { raise }(\varepsilon v) \text { handle } x \succ e \rightarrow e[x \leftarrow \varepsilon v] \text { (handle-all) } \\
& \frac{o \text { escapes } E}{E[o] \rightarrow o} \text { (throw-context) } \frac{e \rightarrow e^{\prime}}{E[e] \rightarrow E\left[e^{\prime}\right]} \text { (context) }
\end{aligned}
$$

Figure 5. Operational semantics of $\lambda_{\mathcal{E}}$

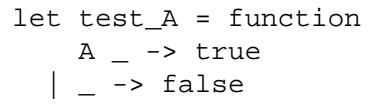

It admits $r^{q} \rightarrow \mathrm{b}^{q(\{A\})}$ (for every $q$ ) as type: the resulting boolean is marked with the union of all security levels attached to the constructor $A$ in the input.

The rotate function performs a transposition of three constructors:

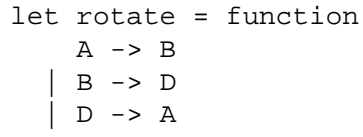

The same transposition arises between the corresponding fields of the types describing the input and the output of this

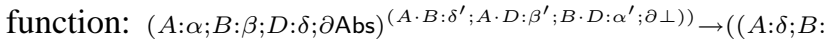
$\alpha ; D: \beta ; \partial \mathrm{Abs})^{\left(A \cdot B: \beta^{\prime} ; A \cdot D: \alpha^{\prime} ; B \cdot D: \delta^{\prime} ; \partial \perp\right)}$. Because our type system guards the type of each branch of the case clause before computing the type of the whole expression, it is able to exactly relate in this example the security levels associated with each pair of constructors of the output with those of the input.

\section{$7 \quad \lambda$-calculus with exceptions}

\subsection{The $\lambda_{\mathcal{E}}$-calculus}

In this section, we define a $\lambda$-calculus with "let" binding and exceptions, $\lambda_{\mathcal{E}}$. Let $\mathcal{E}$ be a denumerable set whose elements are called exception names and denoted by $\varepsilon$. Then values, outcomes, expressions and evaluation contexts of $\lambda_{\mathcal{E}}$ are defined as follows:

$$
\begin{array}{rll}
v & ::= & x|k|(v, v)|\lambda x . e| \varepsilon v \\
o & ::= & v \mid \text { raise }(\varepsilon v) \\
e & ::= & v|v v| \pi_{j} v \mid \text { raise } v \mid \text { let } x=v \text { in } e \mid E[e] \\
E & ::= & \text { bind } x=[] \text { in } e \\
& \text { [] handle } \varepsilon x \succ e \\
& {[] \text { handle } x \succ e}
\end{array}
$$

In fact, $\lambda_{\mathcal{E}}$ is the language studied in [13] (where it is referred to as "Core $M L$ ") deprived of the constructions dealing with references. Its small-step semantics is recalled in figure 5. (o escapes $E$ if and only if $E[o]$ cannot be reduced by one of the rules (bind), (handle) and (handle-all)).

Because of the presence of effects, our presentation of $\lambda_{\mathcal{E}}$ differs from that of $\lambda_{+}$in two points. First, following [20], it is restricted to a call-by-value setting to preserve confluence. Second, we introduce a segregation between values and expressions. This syntactic restriction enables a lighter formulation of the type system and allows it to remain independent of the evaluation order. It does not reduce the expressiveness of the language because usual expressions may be encoded into our restricted syntax in a straightforward manner (see [13], section 5.7).

\subsection{Encoding $\lambda_{\mathcal{E}}$ into $\lambda_{+}^{\mathrm{CBV}}$}

Let the constructors of $\lambda_{+}$be exactly the exception names of $\lambda_{\mathcal{E}}$, with an additional one, denoted by $\eta$ (i.e. $\mathcal{C}=\mathcal{E} \cup\{\eta\})$. The basic idea of the encoding introduced by figure 6 is to translate every expression $e$ of $\lambda_{\mathcal{E}}$ into an expression $\llbracket e \rrbracket$ of $\lambda_{+}$such that if $e$ evaluates to a value $v$ without raising an exception then $\llbracket e \rrbracket$ evaluates to $\eta(v)$ in $\lambda_{+}^{\mathrm{CBV}}$ and if the execution of $e$ raises an exception $\varepsilon v$ then $\llbracket e \rrbracket$ reduces to $\varepsilon(v)$. Such an encoding may be defined in a systematic way using monads $[4,19]$, but here we prefer, for simplicity, a direct translation. It is stable w.r.t. substitution in the sense that $\llbracket e[x \leftarrow v] \rrbracket=\llbracket e \rrbracket[x \Leftarrow(v)]$. The following lemmas establish the correctness of the encoding with respect to the semantics.

Lemma 7.1 (Correctness) If $e \rightarrow e^{\prime}$ then $\llbracket e \rrbracket \rightarrow_{\mathrm{CBV}}^{+} \llbracket e^{\prime} \rrbracket$.

Proof. By induction on the derivation of $e \rightarrow e^{\prime}$.

$\circ$ Case $(\beta) . \quad e$ is $\left(\lambda x . e_{0}\right) v$ and $e^{\prime}$ is $e_{0}[x \leftarrow v]$. Then $\llbracket e \rrbracket=\left(\lambda x . \llbracket e_{0} \rrbracket\right)(v)$. By $(\beta)$, we have $\llbracket e \rrbracket \rightarrow_{\mathrm{CBV}} \llbracket e_{0} \rrbracket[x \Leftarrow$ $(v)]$, i.e. $\llbracket e \rrbracket \rightarrow_{\mathrm{CBV}} \llbracket e^{\prime} \rrbracket$.

$\circ$ Case (proj). $e$ is $\pi_{j}\left(v_{1}, v_{2}\right), e^{\prime}$ is $v_{j}$. Then $\llbracket e \rrbracket=$ $\eta\left(\pi_{j}\left(\left(v_{1}\right),\left(v_{2}\right)\right)\right)$. By (proj), we have $\llbracket e \rrbracket \rightarrow_{\mathrm{CBV}} \eta\left(v_{j}\right)$, i.e. $\llbracket e \rrbracket \rightarrow_{\mathrm{CBV}} \llbracket e^{\prime} \rrbracket$.

$\circ$ Case (bind). $e$ is bind $x=v$ in $e_{0}$ and $e^{\prime}$ is $e_{0}[x \leftarrow v]$. We have $\llbracket e \rrbracket=$ case $\left\{\{\eta\}: y_{1} \mapsto\right.$ let $x=$ $\bar{\eta} y_{1}$ in $\left.\llbracket e_{0} \rrbracket ; \quad \mathcal{E}: y_{1} \mapsto y_{1}\right\}$ Then, by applying successively (case) and (bind), $\llbracket e \rrbracket \rightarrow_{\mathrm{CBV}}^{2} \llbracket e_{0} \rrbracket[x \leftarrow(v)]$, i.e. $\llbracket e \rrbracket \rightarrow_{\mathrm{CBV}}^{2} \llbracket e^{\prime} \rrbracket$. 


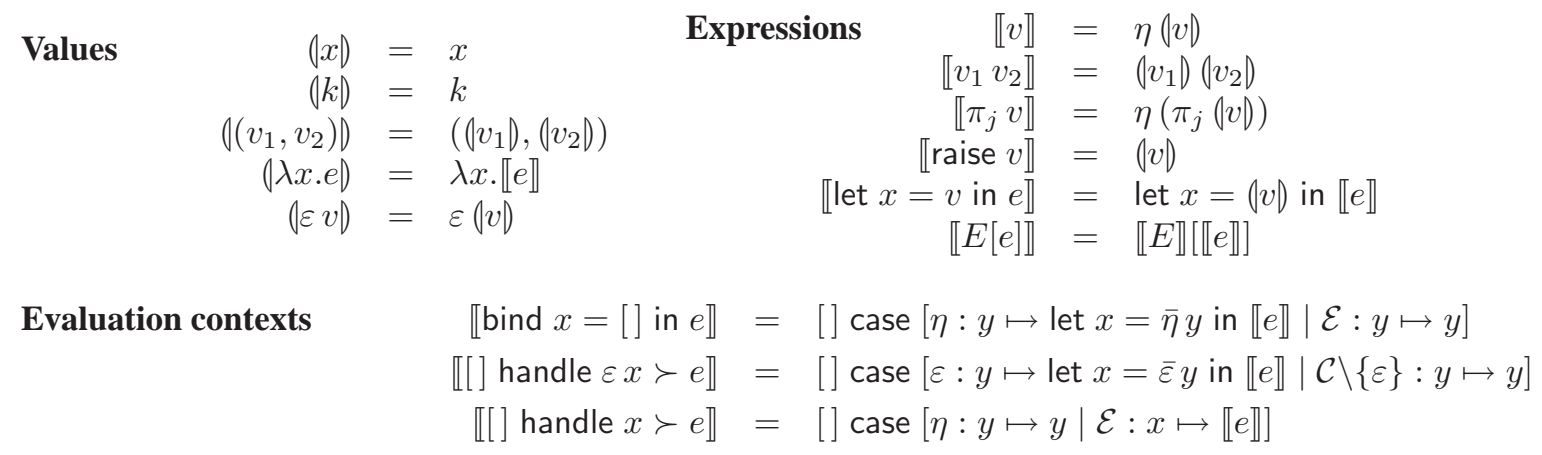

Figure 6. Encoding $\lambda_{\mathcal{E}}$ into $\lambda_{+}$

- Cases (handle), (handle-all) and (throw-context) are similar to (bind).

- Case (context). $\quad e$ is $E\left[e_{0}\right]$ and $e^{\prime}$ is $E\left[e_{0}^{\prime}\right]$ with $e_{0} \rightarrow e_{0}^{\prime}$. By induction hypothesis, we have $\llbracket e_{0} \rrbracket \rightarrow_{\mathrm{CBV}}^{+}$ $\llbracket e_{0}^{\prime} \rrbracket$. Then, by (context), $\llbracket E \rrbracket\left[\llbracket e_{0} \rrbracket\right] \rightarrow_{\mathrm{CBV}}^{+} \llbracket E \rrbracket\left[\llbracket e_{0}^{\prime} \rrbracket\right]$, i.e. $\llbracket e \rrbracket \rightarrow_{\mathrm{CBV}}^{+} \llbracket e^{\prime} \rrbracket$.

Lemma 7.2 (Values) If $\left(v_{1}\right)=\left(v_{2}\right)$ then $v_{1}=v_{2}$.

Proof. By induction on the definition of $(\cdot)$ and $\llbracket \cdot \rrbracket$.

\subsection{Typing $\lambda_{\mathcal{E}}$}

We now define a type system for $\lambda_{\mathcal{E}}$ and prove its correctness by translating each $\lambda_{\mathcal{E}}$ judgement into a $\lambda_{+}$one. The type algebra for $\lambda_{\mathcal{E}}$ is a simple subset of that of $\lambda_{+}$. Restricted types, alternatives, rows and matrices (denoted by bold meta-variables) are defined as follows:

$$
\begin{aligned}
\mathbf{t} & ::=\text { int }^{\ell}|\mathbf{t} \times \mathbf{t}| \mathbf{t} \rightarrow \mathbf{r}^{\mathbf{q}} \mid \dot{\mathbf{r}}^{\dot{\mathbf{q}}} \\
\mathbf{a} & ::=\text { Abs } \mid \text { Pret } \\
\mathbf{r} & ::=\{c \mapsto \mathbf{a}\} \\
\mathbf{q} & ::=\{c \cdot c \mapsto \ell\}
\end{aligned}
$$

The meta-variable $\dot{\mathbf{r}}$ (resp. $\dot{\mathbf{q}}$ ) stands for a row $\mathbf{r}$ (resp. matrix q) carrying no information about $\eta$, i.e. such that $\mathbf{r}(\eta)=$ Abs (resp. $\forall \varepsilon \in \mathcal{E}, \mathbf{q}(\eta \cdot \varepsilon)=\perp$ ). Types for integers and pairs remain unchanged. In this restriction, $\lambda_{+}$sum types play two distinct roles. First they appear as effects on the right-hand-side of function types. Here, they describe the possible normal or exceptional results the function may produce, with associated security levels. Second, they are used to type expressions whose result is an exception: $\dot{\mathbf{r}}{ }^{\dot{\mathrm{q}}}$. There, we use dotted versions of content and level rows to signify that the fields relating to the $\eta$ constructor may be constrained to be Abs and $\perp$, respectively (the translation of an exception value into $\lambda_{+}$is a sum whose head constructor must belong to $\mathcal{E}$ ). $\lambda_{\mathcal{E}}$ 's polytypes (i.e. nonempty upwardclosed sets of types $\mathbf{t}$ ) are denoted by the meta-variable $\mathbf{s}$.

The typing rules for $\lambda_{\mathcal{E}}$ are given in figure 7 . We distinguish two forms of judgements: $\Gamma \vdash v: \mathbf{t}$ and $\Gamma \Vdash e: \mathbf{r}^{\mathbf{q}}$. The former deals with values and involves a simple type (because values cannot raise any exception). The latter associates to an expression an effect $\mathbf{r}^{\mathbf{q}}$, describing what kind of result (value or exceptions) it may produce: $\mathbf{r}(\eta)$ is Abs if $e$ never reduces to a value and is Pre $\mathbf{t}$ if $e$ may produce a value of type $\mathbf{t}$. Analogously, for every expression name $\varepsilon, \mathbf{r}(\varepsilon)$ is Abs if $e$ never raises an exception named $\varepsilon$ and is Pre $\mathbf{t}$ if it may raise such an exception with an argument of type t. $\mathbf{q}\left(c_{1} \cdot c_{2}\right)$ is as expected an approximation of the level of the information one leaks by revealing that $e$ produces a result of name $c_{1}$ (value or exception) rather than $c_{2} . \lambda_{\mathcal{E}}$ 's typing rules may be obtained by a simple combination of $\lambda_{+}$ rules (figure 4$)$ and the translation of $\lambda_{\mathcal{E}}$ into $\lambda_{+}(\llbracket \cdot \rrbracket$ for $\Vdash$ judgements on expressions and $(\cdot)$ for $\vdash$ judgements on values), with some straightforward constraint simplifications. First, in the second premise of E-BIND, we may assume in the right-hand-side of the $\Rightarrow$ that $\mathbf{r}_{1}(\eta)=$ Pre $\mathbf{t}$ for some $\mathbf{t}$ (we write $\overline{\operatorname{Pre}} \mathbf{r}_{1}(\eta)$ for such a $\mathbf{t}$ ). Second, because $\{\eta\}$ and $\mathcal{E}$ are complementary, $\mathbf{q}_{1}(\{\eta\})=\mathbf{q}_{1}(\mathcal{E})$ holds. Therefore, the constraint $\left[\mathbf{q}_{1}(\{\eta\}), \mathbf{q}_{1}(\mathcal{E})\right] \leqslant\left[\left(\mathbf{r}_{1} \mathbf{q}_{1}\right)_{\mid \mathcal{E}}, \mathbf{r}_{2} \mathbf{q}_{2}\right] \leq \mathbf{r}^{\mathbf{q}}$ is simplified into $\mathbf{q}_{1}(\{\eta\}) \triangleq\left[\left(\mathbf{r}_{1} \mathbf{q}_{1}\right)_{\mid \mathcal{E}}, \mathbf{r}_{2} \mathbf{q}_{2}\right] \leq \mathbf{r}^{\mathbf{q}}$. Rules E-HANDLE and E-HANDLEALL present analogous simplifications.

The following lemma states the soundness of this type system by considering each of its judgements as one of $\lambda_{+}$.

Lemma 7.3 (Soundness) $\Gamma \vdash v: \mathbf{t}$ implies $\Gamma \vdash|v\rangle: \mathbf{t}$. $\Gamma \Vdash e: \mathbf{r}^{\mathbf{q}}$ implies $\Gamma \vdash \llbracket e \rrbracket: \mathbf{r}^{\mathbf{q}}$.

From this correspondence, we immediately obtain the following non-interference result for $\lambda_{\mathcal{E}}$. For simplicity, the statement only concerns integer results, but a more general one can be obtained.

Theorem 7.1 (Non-interference) Choose $\ell, h \in \mathcal{L}$ such that $h \not \leq \ell$. Let $h \unlhd\left[t_{1}, t_{2}\right] \leq t$. Assume $(x \mapsto t) \Vdash$ 
Values

$$
\begin{aligned}
& \begin{array}{llllll}
\text { V-INT } & \text { V-VAR } & \text { V-PAIR } & \text { V-ABS } & \text { V-EXN } \\
\Gamma \vdash k: \text { int }^{*} & \frac{\mathbf{t} \in \Gamma(x)}{\Gamma \vdash x: \mathbf{t}} & \frac{\Gamma \vdash v_{1}: \mathbf{t}_{1}}{\Gamma \vdash v_{2}: \mathbf{t}_{2}} & \frac{\Gamma[x \mapsto \mathbf{t}] \Vdash e: \mathbf{r}^{\mathbf{q}}}{\Gamma \vdash\left(v_{1}, v_{2}\right): \mathbf{t}_{1} \times \mathbf{t}_{2}} & \frac{\Gamma \vdash v: \mathbf{t}}{\Gamma \vdash \lambda x \cdot e: \mathbf{t} \rightarrow \mathbf{r}^{\mathbf{q}}} & \frac{\Gamma \vdash \varepsilon v:(\varepsilon: \operatorname{Pret} ; *)^{*}}{\Gamma \vdash \varepsilon}
\end{array} \\
& \frac{\mathrm{V}-\mathrm{SUB}}{\Gamma \vdash v: \mathbf{t}^{\prime} \quad \mathbf{t}^{\prime} \leq \mathbf{t}}
\end{aligned}
$$

\section{Expressions}

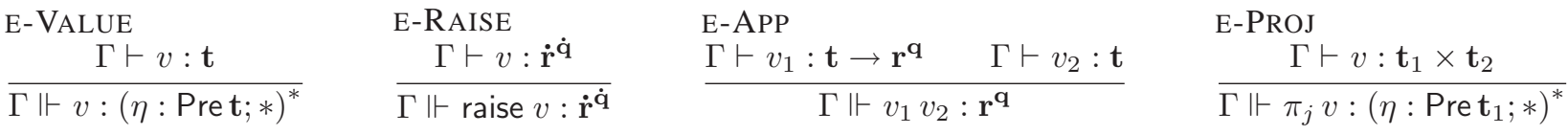

$$
\begin{aligned}
& \text { E-BIND } \\
& \frac{\Gamma \Vdash e_{1}: \mathbf{r}_{1} \mathbf{q}_{1} \quad \mathbf{r}_{1} \cap\{\eta\} \Rightarrow \Gamma\left[x \mapsto \overline{\operatorname{Pre}} \mathbf{r}_{1}(\eta)\right] \Vdash e_{2}: \mathbf{r}_{2}{ }^{\mathbf{q}_{2}} \quad \mathbf{q}_{1}(\{\eta\}) \Vdash\left[\left(\mathbf{r}_{1}{ }^{\mathbf{q}_{1}}\right)_{\mid \mathcal{E}}, \mathbf{r}_{2}{ }^{\mathbf{q}_{2}}\right] \leq \mathbf{r}^{\mathbf{q}}}{\Gamma \Vdash \operatorname{bind} x=e_{1} \text { in } e_{2}: \mathbf{r}^{\mathbf{q}}} \\
& \text { E-HANDLE } \\
& \frac{\Gamma \Vdash e_{1}: \mathbf{r}_{1}{ }^{\mathbf{q}_{1}} \quad \mathbf{r}_{1} \text { ก }\{\varepsilon\} \Rightarrow \Gamma\left[x \mapsto \overline{\operatorname{Pre}} \mathbf{r}_{1}(\varepsilon)\right] \Vdash e_{2}: \mathbf{r}_{2}{ }^{\mathbf{q}_{2}} \quad \mathbf{q}_{1}(\{\varepsilon\}) \triangleq\left[\left(\mathbf{r}_{1}{ }^{\mathbf{q}_{1}}\right)_{\mid \mathcal{C} \backslash\{\varepsilon\}}, \mathbf{r}_{2}{ }^{\mathbf{q}_{2}}\right] \leq \mathbf{r}^{\mathbf{q}}}{\Gamma \Vdash e_{1} \text { handle } \varepsilon x \succ e_{2}: \mathbf{r}^{\mathbf{q}}} \\
& \text { E-HANDLEALL } \\
& \frac{\Gamma \Vdash e_{1}: \mathbf{r}_{1} \mathbf{q}_{1} \quad \mathbf{r}_{1} \cap \mathcal{E} \Rightarrow \Gamma\left[x \mapsto\left(\mathbf{r}_{1}{ }^{\mathbf{q}_{1}}\right)_{\mid \mathcal{E}}\right] \Vdash e_{2}: \mathbf{r}_{2}{ }^{\mathbf{q}_{2}} \quad \mathbf{q}_{1}(\{\eta\}) \Vdash\left[\left(\mathbf{r}_{1}{ }^{\mathbf{q}_{1}}\right)_{\mid\{\eta\}}, \mathbf{r}_{2}{ }^{\mathbf{q}_{2}}\right] \leq \mathbf{r}^{\mathbf{q}}}{\Gamma \Vdash e_{1} \text { handle } x \succ e_{2}: \mathbf{r}^{\mathbf{q}}} \\
& \text { E-LET } \\
& \frac{\Gamma \vdash v: \mathbf{s} \quad \Gamma[x \mapsto \mathbf{s}] \Vdash e: \mathbf{r}^{\mathbf{q}}}{\Gamma \Vdash \text { let } x=v \text { in } e: \mathbf{r}^{\mathbf{q}}} \\
& \frac{\Gamma \Vdash e: \mathbf{r}^{\prime \mathbf{q}^{\prime}} \quad \mathbf{r}^{\prime} \leq \mathbf{r} \quad \mathbf{q}^{\prime} \leq \mathbf{q}}{\Gamma \Vdash e: \mathbf{r}^{\mathbf{q}}}
\end{aligned}
$$

\section{Figure 7. The type system for $\lambda_{\mathcal{E}}$}

$e:\left(\eta: \text { Pre int }{ }^{\ell} ; *\right)^{*}$, where e is a $\lambda_{\mathcal{E}}$ expression. If $\vdash v_{i}: t_{i}$ and $e\left[x \Leftarrow v_{i}\right] \rightarrow^{\star} v_{i}$, for $i \in\{1,2\}$, then $v_{1}=v_{2}$.

Proof. By lemmas 7.1, 7.2, 7.3 and theorem 4.2.

\section{One-dimensional systems}

We now present two type systems, $\lambda_{+}^{(1)}$ and $\lambda_{\mathcal{E}}^{(1)}$, derived from $\lambda_{+}$and $\lambda_{\mathcal{E}}$. Although they provide a less accurate analysis, they remain of interest because they involve simpler annotations and give us the opportunity to compare our treatment of exceptions with previous works [5, 13].

\subsection{The system $\lambda_{+}^{(1)}$}

In this section, we present a more lightweight type system for $\lambda_{+}$(which we will refer to as $\lambda_{+}^{(1)}$ ) where information carried by a sum is described by a one-dimensional row of levels indexed by constructor names. We begin by restricting the case construction of $\lambda_{+}$to have only two handlers: $e$ case $\left[\{c\}: x \mapsto e_{1} \mid(\mathcal{C} \backslash\{c\}): x \mapsto e_{2}\right]$. (Note that such a restriction still allows multiple matching by nesting case clauses. Moreover, it still allows the encoding of exceptions into sums, see section 8.2.)

The point of this restriction is that the use of matrices $q$ in the CASE rule of $\lambda_{+}$is now limited to an access of the form $q(\{c\})$ for some $c \in \mathcal{C}$ (or $q(\mathcal{C} \backslash\{c\})$ which is equal to $q(\{c\}))$. The basic idea of $\lambda_{+}^{(1)}$ consists in directly storing these levels in sum types, rather than the whole matrix $q$. Thus, types, alternatives, rows and vectors of $\lambda_{+}^{(1)}$ (which are denoted $\bar{t}, \bar{a}, \bar{r}$ and $\bar{q}$, respectively) are obtained by the same grammar as that of section 4.1, where the definition of matrices is replaced by $\bar{q}::=\{c \mapsto \ell\}$. Sum types now have the form $\bar{r} \bar{q}$ where both $\bar{r}$ and $\bar{q}$ are indexed by $\mathcal{C}$. The meaning of $\bar{r}$ remains unchanged. Simultaneously, the vector $\bar{q}$ indicates for each constructor $c$ how much information 


$$
\begin{aligned}
& \operatorname{CASE}^{(1)} \\
& \quad \Gamma \vdash e: \bar{r}^{\bar{q}} \quad \Gamma\left[x_{1} \mapsto\left(\bar{r}^{\bar{q}}\right)_{\mid\{c\}}\right] \vdash e_{1}: \bar{t} \\
& \quad \frac{\Gamma\left[x_{2} \mapsto\left(\bar{r}^{\bar{q}}\right)_{\mid \mathcal{C} \backslash\{c\}} \vdash \vdash e_{2}: \bar{t} \quad \bar{q}(c) \triangleleft \bar{t}\right.}{\Gamma \vdash v \text { case }\left[\{c\}: x_{1} \mapsto e_{1} \mid \mathcal{C} \backslash\{c\}: x_{2} \mapsto e_{2}\right]: \bar{t}}
\end{aligned}
$$

\section{Figure 8. The $\mathrm{CASE}^{(1)}$ rule of $\lambda_{+}^{(1)}$}

Types

$$
\begin{array}{cc}
\frac{\ell \leq \ell^{\prime}}{\ell \triangleleft \text { int }^{\ell^{\prime}}} \quad \frac{\ell \triangleleft \bar{t}}{\ell \triangleleft \bar{t}^{\prime} \rightarrow \bar{t}} & \frac{\ell \triangleleft \bar{t}_{1} \quad \ell \triangleleft \bar{t}_{2}}{\ell \triangleleft \bar{t}_{1} \times \bar{t}_{2}} \\
\ell \triangleleft \bar{r} & \ell \triangleleft \bar{r} \\
\frac{\forall c, \bar{r}(c) \neq \mathrm{Abs} \Rightarrow \ell \leq \bar{q}(c)}{\ell \triangleleft \bar{r}^{\bar{q}}} & \frac{\bar{r}=(*: \operatorname{Pre} * ; \partial \mathrm{Abs})}{\ell \triangleleft \bar{r}^{\bar{q}}}
\end{array}
$$

\section{Rows}

$$
\ell \triangleleft \operatorname{Abs} \quad \frac{\ell \triangleleft \bar{t}}{\ell \triangleleft \operatorname{Pre} \bar{t}} \quad \frac{\forall c \in \mathcal{C}, \ell \triangleleft \bar{r}(c)}{\ell \triangleleft \bar{r}}
$$

Figure 9. One-dimensional guards

one may leak by testing whether the expression at hand produces a result whose head constructor is $c$. This corresponds to the level given by $q(\{c\})=\sqcup\left\{q\left(c \cdot c^{\prime}\right) \mid c^{\prime} \neq c\right\}$ in the previous system. In other words, a matrix $q$ is approximated in $\lambda_{+}^{(1)}$ by the vector $\bar{q}$ defined by $\bar{q}(c)=q(\{c\})$.

This correspondence allows us to derive the typing rules of $\lambda_{+}^{(1)}$ from those of $\lambda_{+}$. All the rules remain syntactically unchanged, except CASE whose new version, $\operatorname{CASE}^{(1)}$, is given in figure 8 . The main difference with the previous rule lies in the fact that the union of the resulting types of the two branches is computed before marking it by the level $\bar{q}(c)$ : the type system is no longer able to take into account the origin of each component of the resulting type while guarding it. As a result, the predicate $\triangleleft$ in $\lambda_{+}^{(1)}$, defined in figure 9, takes only two arguments: a security level and a type. $\ell \triangleleft \bar{r}^{\bar{q}}$ constrains each field of $\bar{q}$ corresponding to an entry of $\bar{r}$ which is Pre $*$ to be $\ell$ or greater. There is nevertheless a special case: if all but one fields of $\bar{r}$ are Abs, (that means when the possible head constructor the expression may produce is known by the type system) then it is unnecessary to constrain the row $\bar{q}$.

We now briefly prove the correctness of $\lambda_{+}^{(1)}$ thanks to an encoding into $\lambda_{+}$. We introduce a mapping $\left\langle\langle\cdot\rangle\right.$ from $\lambda_{+}^{(1)}$ types into those of $\lambda_{+}$. It is a homomorphism, except on vectors which are translated into matrices using the following approximation: $\langle\bar{q}\rangle\left(c_{1} \cdot c_{2}\right)=\bar{q}\left(c_{1}\right) \sqcap \bar{q}\left(c_{2}\right)$. We extend $\langle\cdot\rangle\rangle$ pointwise on polytypes and environments.

Lemma 8.1 (Subtyping) If $\bar{t}_{1} \leq \bar{t}_{2}$ then $\left\langle\left\langle\bar{t}_{1}\right\rangle \leq\left\langle\bar{t}_{2}\right\rangle\right.$.

Proof. We first prove that $\bar{q}_{1} \leq \bar{q}_{2}$ implies $\left\langle\left\langle\bar{q}_{1}\right\rangle \leq\left\langle\left\langle\bar{q}_{2}\right\rangle\right.\right.$. Assume $\bar{q}_{1} \leq \bar{q}_{2}$. Let $c$ and $c^{\prime}$ be two distinct constructor names. We have $\bar{q}_{1}(c) \leq \bar{q}_{2}(c)$ and $\bar{q}_{1}\left(c^{\prime}\right) \leq \bar{q}_{2}\left(c^{\prime}\right)$. This yields $\bar{q}_{1}(c) \sqcap \bar{q}_{1}\left(c^{\prime}\right) \leq \bar{q}_{2}(c) \sqcap \bar{q}_{2}\left(c^{\prime}\right)$, i.e. $\left\langle\bar{q}_{1}\right\rangle\left(c \cdot c^{\prime}\right) \leq$ $\left\langle\left\langle\bar{q}_{2}\right\rangle\right\rangle\left(c \cdot c^{\prime}\right)$.

Conclude by induction on the structure of types, alternatives and rows.

\section{Lemma 8.2 (Guard) $\ell \triangleleft \bar{t}$ implies $\ell \leqslant[\langle\bar{t}\rangle,\langle\langle\bar{t}\rangle]$.}

Proof. By induction on the structure of $\bar{t}$. The only case of interest is $\bar{t}=\bar{r}^{\bar{q}}$. Let $\left.r^{q}=\langle\bar{r}\rangle\right\rangle^{\langle\bar{q}\rangle}=\langle\langle\bar{r} \bar{q}\rangle$. By definition, $\ell \triangleleft \bar{r}^{\bar{q}}$ implies $\ell \triangleleft \bar{r}$. By induction hypothesis and GUARDS-Row, it follows that $\ell \unlhd[r, r]$. If only one entry of $\bar{r}$ is Pre then the same arises for $r$ and $\ell \leqslant\left[r^{q}, r^{q}\right]$. Otherwise, if $r\left(c_{1}\right)=$ Pre $*$ and $r\left(c_{2}\right)=$ Pre $*$ for some $c_{1} \neq c_{2}, \ell \triangleleft \bar{r} \bar{q}$ implies $\ell \leq \bar{q}\left(c_{1}\right)$ and $\ell \leq \bar{q}\left(c_{2}\right)$. It follows $\ell \leq \bar{q}\left(c_{1}\right) \sqcap \bar{q}\left(c_{2}\right)=q\left(c_{1} \cdot c_{2}\right)$. Once again, we conclude that $\ell \leqslant\left[r^{q}, r^{q}\right]$.

Lemma 8.3 (Restriction) $\left\langle\left(\bar{r}^{\bar{q}}\right)_{\mid C}\right\rangle=\left\langle\left\langle\bar{r}^{\bar{q}}\right\rangle_{\mid C}\right.$ holds for all $C \subseteq \mathcal{C}$.

Proof. It is clear that $\left\langle\left\langle\bar{r}_{\mid C}\right\rangle\right\rangle=\langle\bar{r}\rangle_{\mid C}$. Let us consider $c_{1} \neq$ $c_{2}$. If $c_{1} \in C$ and $c_{2} \in C$ (i.e. $\left.c_{1} \cdot c_{2} \in C^{2}\right)$ then $\langle\bar{q}\rangle_{C^{2}}\left(c_{1}\right.$. $\left.c_{2}\right)=\langle\bar{q}\rangle\left(c_{1} \cdot c_{2}\right)=\bar{q}\left(c_{1}\right) \sqcap \bar{q}\left(c_{2}\right)=\bar{q}_{\mid C}\left(c_{1}\right) \sqcap \bar{q}_{\mid C}\left(c_{2}\right)=$ $\left\langle\left\langle\bar{q}_{\mid C}\right\rangle\left(c_{1} \cdot c_{2}\right)\right.$. Otherwise, either $\bar{q}_{\mid C}\left(c_{1}\right)$ or $\bar{q}_{\mid C}\left(c_{2}\right)$ is $\perp$. Then $\left.\left\langle\bar{q}_{\mid C}\right\rangle\right\rangle\left(c_{1} \cdot c_{2}\right)=\bar{q}_{\mid C}\left(c_{1}\right) \sqcap \bar{q}_{\mid C}\left(c_{2}\right)=\perp=\left\langle\langle\bar{q}\rangle_{\mid C^{2}}\left(c_{1}\right.\right.$. $\left.c_{2}\right)$. We conclude that $\left\langle\langle\bar{q}\rangle_{\mid C^{2}}=\left\langle\left\langle\bar{q}_{\mid C}\right\rangle\right\rangle\right.$.

Lemma 8.4 (Soundness) If $\Gamma \vdash e: \bar{t}$ then $\langle\langle\Gamma\rangle \vdash e:\langle\bar{t}\rangle$.

Proof. By induction on the derivation of $\Gamma \vdash e: \bar{t}$.

$\circ$ Case $\mathrm{CASE}^{(1)} . e$ is $e^{\prime}$ case $\left[\{c\}: x_{1} \mapsto e_{1}\right.$ $\left.\mathcal{C} \backslash\{c\}: x_{2} \mapsto e_{2}\right]$. Among $\operatorname{CASE}^{(1)}$ 's premises we find $\Gamma \vdash e^{\prime}: \bar{r}^{\bar{q}}, \Gamma\left[x_{1} \mapsto\left(\bar{r}^{\bar{q}}\right)_{\mid\{c\}}\right] \vdash e_{1}: \bar{t}, \Gamma\left[x_{2} \mapsto\right.$ $\left.\left(\bar{r}^{\bar{q}}\right)_{\mid \mathcal{C} \backslash\{c\}}\right] \vdash e_{2}: \bar{t}$ and $\bar{q}(c) \triangleleft \bar{t}$. Let $r^{q}=\langle\langle\bar{r}\rangle\langle\bar{q}\rangle=$ $\left\langle\left\langle\bar{r}^{\bar{q}}\right\rangle\right.$. By induction hypothesis and lemma 8.3, we obtain $\left.\langle\Gamma\rangle \vdash e^{\prime}:\langle\bar{r}\rangle\right\rangle^{\langle\bar{q}\rangle},\left\langle\langle\Gamma\rangle\left[x_{1} \mapsto\left(\langle\bar{r}\rangle^{\langle\bar{q}\rangle}\right)_{\mid\{c\}}\right] \vdash e_{1}:\right.$ $\langle\bar{t}\rangle, \Gamma\left[x_{2} \mapsto(\langle\bar{r}\rangle\langle\bar{q}\rangle)_{\mid \mathcal{C} \backslash\{c\}}\right] \vdash e_{2}:\langle\langle\bar{t}\rangle\rangle$. Moreover $\left\langle\langle\bar{q}\rangle(\{c\})=\left\langle\langle\bar{q}\rangle(\mathcal{C} \backslash\{c\})=\sqcup\left\{\bar{q}(c) \sqcap \bar{q}\left(c^{\prime}\right) \mid c^{\prime} \neq c\right\}\right.\right.$. Then $\langle\bar{q}\rangle(\{c\}) \leq \bar{q}(c)$ and $\langle\langle\bar{q}\rangle(\mathcal{C} \backslash\{c\}) \leq \bar{q}(c)$. By lemmas 8.2 and $4.2, \bar{q}(c) \triangleleft \bar{t}$ yields $[\langle\bar{q}\rangle(\{c\}),\langle\langle\bar{q}\rangle(\mathcal{C} \backslash\{c\})] \preccurlyeq$ $[\langle\bar{t}\rangle,\langle\langle\bar{t}\rangle] \leq\langle\langle\bar{t}\rangle$. Conclude by an instance of CASE.

$\circ$ Case SuB ${ }^{(1)}$. By lemma 8.1 and the induction hypothesis.

Other cases are immediate. 


$$
\begin{aligned}
& \text { E-BIND }{ }^{(1)} \\
& \Gamma \Vdash e_{1}: \overline{\mathbf{r}}_{1}^{\overline{\mathbf{q}}_{1}} \quad \Gamma\left[x \mapsto \overline{\operatorname{Pre}} \overline{\mathbf{r}}_{1}(\eta)\right] \Vdash e_{2}: \overline{\mathbf{r}}_{2}^{\overline{\mathbf{q}}_{2}} \\
& \left(\overline{\mathbf{r}}_{1}^{\overline{\mathbf{q}}_{1}}\right)_{\mid \mathcal{E}} \leq \overline{\mathbf{r}}^{\overline{\mathbf{q}}} \quad \overline{\mathbf{q}}(\eta) \triangleleft \overline{\mathbf{r}}^{\overline{\mathbf{q}}} \\
& \Gamma \Vdash \text { bind } x=e_{1} \text { in } e_{2}: \overline{\mathbf{r}}^{\overline{\mathbf{q}}} \\
& \text { E-HANDLE }{ }^{(1)} \\
& \Gamma \Vdash e_{1}: \overline{\mathbf{r}}_{1}^{\overline{\mathbf{q}}_{1}} \quad \Gamma\left[x \mapsto \overline{\operatorname{Pre}} \overline{\mathbf{r}}_{1}(\varepsilon)\right] \Vdash e_{2}: \overline{\mathbf{r}}^{\overline{\mathbf{q}}} \\
& \left(\overline{\mathbf{r}}_{1}^{\overline{\mathbf{q}}_{1}}\right)_{\mid \mathcal{C} \backslash\{\varepsilon\}} \leq \overline{\mathbf{r}}^{\overline{\mathbf{q}}} \quad \overline{\mathbf{q}}(\varepsilon) \triangleleft \overline{\mathbf{r}}^{\overline{\mathbf{q}}} \\
& \Gamma \Vdash e_{1} \text { handle } \varepsilon x \succ e_{2}: \overline{\mathbf{r}}^{\overline{\mathbf{q}}} \\
& \text { E-HANDLEALL }{ }^{(1)} \\
& \Gamma \Vdash e_{1}: \overline{\mathbf{r}}_{1}^{\overline{\mathbf{q}}_{1}} \quad \Gamma\left[x \mapsto\left(\overline{\mathbf{r}}_{1}^{\overline{\mathbf{q}}_{1}}\right)_{\mid \mathcal{E}}\right] \Vdash e_{2}: \overline{\mathbf{r}}^{\overline{\mathbf{q}}} \\
& \left(\overline{\mathbf{r}}_{1}^{\overline{\mathbf{q}}_{1}}\right)_{\mid\{\eta\}} \leq \overline{\mathbf{r}}^{\overline{\mathbf{q}}} \quad \overline{\mathbf{q}}(\eta) \triangleleft \overline{\mathbf{r}}^{\overline{\mathbf{q}}} \\
& \Gamma \Vdash e_{1} \text { handle } x \succ e_{2}: \overline{\mathbf{r}}^{\overline{\mathbf{q}}}
\end{aligned}
$$

\section{Figure 10. The type system for $\lambda_{\mathcal{E}}^{(1)}$}

Theorem 8.1 (Non-interference) Choose $\ell, h \in \mathcal{L}$ such that $h \not \leq \ell$. Let $h \triangleleft t$. Assume $(x \mapsto t) \vdash e$ : int ${ }^{\ell}$, where $e$ is a $\lambda_{+}$expression. If $\vdash e_{i}: t$ and $e\left[x \Leftarrow e_{i}\right] \rightarrow^{\star} k_{i}$, for $i \in\{1,2\}$, then $k_{1}=k_{2}$.

Proof. By lemmas 8.2, 8.4 and theorem 4.2.

\subsection{The $\operatorname{system} \lambda_{\mathcal{E}}^{(1)}$}

Using the same mechanism, it is possible to obtain the corresponding type system $\lambda_{\mathcal{E}}^{(1)}$ for $\lambda_{\mathcal{E}}$. Those of its rules that are different from $\lambda_{\mathcal{E}}$ 's are given in figure 10.

$\lambda_{\mathcal{E}}^{(1)}$ provides a treatment of exceptions that is very similar to that of JFlow $[6,5]$, although the presentation is superficially different. Indeed, JFlow introduces a notion of path labels. Setting aside Java-specific features, paths in JFlow are $\underline{n}$ (which represents normal termination) and names of classes that inherit from Throwable, i.e. classes representing exceptions. This directly corresponds in our framework to $\eta$ and the exception names $\varepsilon \in \mathcal{E}$, respectively. A path label $X$ maps each path $s$ to either the special constant $\underline{\emptyset}$, if the expression cannot terminate through the path $s$, or a security level approximating how much information will be obtained by observing that this path is the effective termination path. This is comparable to our alternatives Abs and Pre $\ell$. The accuracy provided by the $\triangleleft$ constraint when all fields but one of a row are Abs is obtained in JFlow by a non-syntax-directed rule, called single-path rule, allowing $X[\underline{n}]$ to be reset to $\underline{\emptyset}$ if all other paths are already mapped to $\underline{\emptyset}$ by $X$. (Because exception names are classes in Java and are therefore equipped with subtyping, this rule cannot be applied safely to non- $\underline{n}$ paths. But, as noticed by Myers, if exceptions were not identified with classes, the single-path rule could be applied to exceptions too.)

If we constrain the field $\eta$ of every row $r$ of $\lambda_{\mathcal{E}}^{(1)}$ to be Pre $*$, we obtain a treatment of exceptions similar to that proposed in [13]. Then, every lower-bound constraint on the $\eta$ entry of a row $\bar{q}$ (generated by a $\triangleleft$ constraint) must also constrain some other field of the same row. As a result, it is possible to enforce the invariant that, for any row $\overline{\mathbf{q}}, \overline{\mathbf{q}}(\eta)=$ $\sqcup\{\overline{\mathbf{q}}(\varepsilon) \mid \varepsilon \in \mathcal{E}\}$. The main interest of this policy lies in the fact that, because $\ell \triangleleft(\eta \text { : Pre } t ; \overline{\mathbf{r}})^{\overline{\mathbf{q}}}$ becomes equivalent to $\ell \triangleleft t \wedge \forall \varepsilon,(\overline{\mathbf{r}}(\varepsilon) \neq \mathrm{Abs} \Rightarrow \ell \leq \overline{\mathbf{q}}(\varepsilon))$, the system requires only a very simple form of conditional constraints.

\section{Conclusion}

It is an interesting question in what context this analysis would be useful. Because of the structure of security annotations involving matrices of levels, a type inference algorithm based on our framework is likely to produce very verbose type schemes. That is the reason why it seems difficult to use it as the basis for a generic secure programming language, as we aim at with MLIF [13]. Nevertheless such an implementation might be of interest for automated analysis of very sensitive (relatively to information flow) part of programs for which systems such as $[5,13]$ remain too approximative. Such a case may particularly arise in programming languages for devices with limited ressources, such as JavaCard, where integer constants are used as flags in order to represent different data in an unstructured manner.

Moreover, it seems possible to design a reasonably efficient algorithm addressing type inference and constraint solving for this system: we believe that this is mainly an implementation and proof issue.

Another topic of interest lies in adding mutable cells (a.k.a. references) to $\lambda_{+}$and $\lambda_{\mathcal{E}}$. Obtaining a treatment of references similar to that of [13] (where reference types $t$ ref $^{\ell}$ have an invariant argument $t$ describing the content of the cell and carry an external security annotation $\ell$ related to the reference's identity) is straightforward. This remains an orthogonal problem to the accurate treatment of union types. It mainly requires adding an extra security annotation $p c$ on every typing judgement and on $\rightarrow$ types. Nevertheless, such a framework does not provide as fine-grained a treatment of information flow generated by side-effects as that for functional aspects of the language. For instance, one may rewrite our first example in an imperative style

$$
\begin{aligned}
& \text { if } x \text { then (if } y \text { then } t:=A \text { else } t:=B \text { ) } \\
&\text { else (if } z \text { then } t:=A \text { else } t:=D) ; \\
& \text { let } u=! t \text { case }[A, B \mapsto 1 \mid D \mapsto 0]
\end{aligned}
$$

Then, the system would no longer be able to detect the absence of dependency between $u$ and the value stored in $t t$ 
because in all the branches of this program the content of the cell $t$ must have the same type (i.e. $\left.(A, B, D: \text { Pre } *)^{q}\right)$. Therefore, the three security levels of the matrix $q$ will be constrained to exceed that of $x, y$ and $z$. Thus, an interesting direction for further work consists in obtaining a finegrained analysis of dependencies due to side-effects.

\section{References}

[1] M. Abadi, A. Banerjee, N. Heintze, and J. G. Riecke. A core calculus of dependency. In Conference Record of the 26th ACM Symposium on Principles of Programming Languages, pages 147-160, San Antonio, Texas, Jan. 1999. ACM Press. URL: http://www. soe.ucsc.edu/ abadi/ Papers/flowpopl.ps.

[2] D. E. Denning and P. J. Denning. Certification of programs for secure information flow. Communications of the ACM, 20(7):504-513, July 1977.

[3] N. Heintze and J. G. Riecke. The SLam calculus: Programming with secrecy and integrity. In Conference Record of the 25th ACM Symposium on Principles of Programming Languages, pages 365-377, San Diego, California, Jan. 1998. URL: http://cm.bell-labs.com/cm/cs/who/ nch/slam.ps.

[4] E. Moggi. An abstract view of programming languages. Technical Report ECS-LFCS-90-113, University of Edinburgh, June 1989. URL: http://www.disi.unige.it/ person/MoggiE/ftp/abs-view.ps.gz.

[5] A. C. Myers. JFlow: practical mostly-static information flow control. In Proceedings of the 26th ACM SIGPLANSIGACT on Principles of Programming Languages, pages 228-241, San Antonio, Texas, Jan. 1999. ACM Press. URL: http://www.cs.cornell.edu/andru/papers / popl99/myers-popl99.ps.gz.

[6] A. C. Myers. Mostly-Static Decentralized Information Flow Control. PhD thesis, Massachusetts Institute of Technology, Jan. 1999. Technical Report MIT/LCS/TR-783. URL: http://www.cs.cornell.edu/andru/release/ tr783.ps.gz.

[7] M. Odersky, M. Sulzmann, and M. Wehr. Type inference with constrained types. Theory and Practice of Object Systems, 5(1):35-55, 1999. URL: http://www.cs.mu.oz. au/ sulzmann/publications/tapos.ps.

[8] J. Palsberg and P. Ørbæk. Trust in the $\lambda$-calculus. Lecture Notes in Computer Science, 983:314-330, 1995. URL: ftp://ftp.daimi.au.dk/pub/empl/poe/ lambda-trust.dvi.gz.

[9] J. Palsberg, M. Wand, and P. M. O'Keefe. Type inference with non-structural subtyping. Formal Aspects of Computing, 9:49-67, 1997. URL: http://www.cs .purdue.edu/ homes/palsberg/paper/fac97.ps.gz.

[10] F. Pottier. Wallace: an efficient implementation of type inference with subtyping, Feb. 2000. URL: http://pauillac. inria.fr/ fpottier/wallace/.

[11] F. Pottier. A semi-syntactic soundness proof for $\operatorname{HM}(X)$. Research Report 4150, INRIA, Mar. 2001. URL: ftp://ftp.inria.fr/INRIA/publication/RR/ RR-4150.ps.gz.
[12] F. Pottier and S. Conchon. Information flow inference for free. In Proceedings of the the Fifth ACM SIGPLAN International Conference on Functional Programming (ICFP'00), pages 46-57, Sept. 2000. URL: http://pauillac.inria.fr/ fpottier/publis/ fpottier-conchon-icfp00.ps.gz.

[13] F. Pottier and V. Simonet. Information flow inference for ML. In Proceedings of the 29th ACM Symposium on Principles of Programming Languages (POPL'02), pages 319-330, Portland, Oregon, Jan. 2002. URL: http://cristal.inria.fr/ simonet/publis/ fpottier-simonet-popl02.ps.gz.

[14] D. Rémy. Records and variants as a natural extension of ML. In Proceedings of the Sixteenth Annual Symposium on Principles Of Programming Languages (POPL'89), pages 77-88, Austin, Texas, jan 1989.

[15] D. Rémy. Algèbres Touffues. Application au Typage Polymorphe des Objets Enregistrements dans les Langages Fonctionnels. Thèse de doctorat, Université de Paris 7, 1990. URL: ftp://ftp.inria.fr/INRIA/Projects/ cristal/Didier. Remy/these.ps.gz.

[16] V. Simonet. Fine-grained information flow analysis for a $\lambda$-calculus with sum types. Full version. URL: http://cristal.inria.fr/ simonet/publis/ simonet-csfw-02-long.ps.gz, Feb. 2002.

[17] M. Sulzmann. A general framework for Hindley/Milner type systems with constraints. $\mathrm{PhD}$ thesis, Yale University, Department of Computer Science, May 2000. URL: http://www.cs.mu.oz.au/ sulzmann/ publications/diss.ps.gz.

[18] D. Volpano and G. Smith. A type-based approach to program security. Lecture Notes in Computer Science, 1214:607-621, Apr. 1997. URL: http://www.cs.nps.navy.mil/people/faculty/ volpano/papers/tapsoft97.ps.z.

[19] P. Wadler. Comprehending monads. Mathematical Structures in Computer Science, 2:461-493, 1992. URL: http://cm.bell-labs.com/cm/cs/who/wadler/ papers/monads/monads.ps.gz.

[20] A. K. Wright and M. Felleisen. A syntactic approach to type soundness. Information and Computation, 115(1):38-94, Nov. 1994. URL: http://www.cs.rice.edu/CS/PLT/ Publications/ic94-wf.ps.gz.

[21] S. Zdancewic and A. C. Myers. Secure information flow and CPS. In D. Sands, editor, Proceedings of the 2001 European Symposium on Programming (ESOP'01), Lecture Notes in Computer Science. Springer Verlag, Apr. 2001. URL: http: //www.cs.cornell.edu/zdance/lincont.ps. 